J. DIFFERENTIAL GEOMETRY

$69(2005) \quad 1-42$

\title{
SIMPLE SINGULARITIES AND SYMPLECTIC FILLINGS
}

\author{
Hiroshi Ohta \& KaOru Ono \\ Dedicated to Professor Kyoji Saito on the occasion of his sixtieth birthday
}

\begin{abstract}
It is proved that the diffeomorphism type of the minimal symplectic fillings of the link of a simple singularity is unique. In the proof, the uniqueness of the diffeomorphism type of $\mathbf{C} P^{2} \backslash D$, where $D$ is a pseudo holomorphic rational curve with one $(2,3)$ cusp, is discussed.
\end{abstract}

\section{Introduction}

The link $L$ of an isolated singularity $O$ on an algebraic variety $V$ carries a natural contact structure given by the maximal complex tangency $\xi$. Associated to $(V, O)$, we have two kinds of objects. One is a resolution of the singularity $(V, O)$ and the other is so-called the Milnor fiber, which exists when $(V, O)$ has a smoothing. Both of them provide examples of compact manifolds bounding $L$. From symplectic viewpoint, their boundaries are "symplectically convex". Such manifolds are called symplectic fillings of $(L, \xi)$. In general, the two symplectic fillings produced by the minimal resolution and the Milnor fiber (if it exists) of an isolated singularity on a complex surface have different topologies. In a previous paper [27], we studied topological constraints for minimal symplectic fillings of links of simple singularities on complex surfaces. Here "minimal" means that the symplectic 4-manifold does not contain any symplectically embedded sphere of self-intersection number -1 , which we may call a symplectic (-1)-curve. For simple singularities, Brieskorn

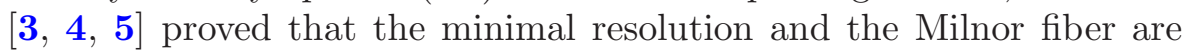
diffeomorphic as a consequence of existence of simultaneous resolution.

The first author is partially supported by Grant-in-Aid for Scientific Research Nos. 12640066 and 15340020, the Ministry of Education, Science, Sports and Culture, Japan. The second author is partially supported by Grant-in-Aid for Scientific Research Nos. 11440015 and 14003419, the Ministry of Education, Science, Sports and Culture, Japan.

Received 06/09/2003. 
Of course, we note that they are not isomorphic as complex manifolds. This result suggests to us that, in these cases, the diffeomorphism type of minimal symplectic fillings should be unique. The goal of this paper is to prove the following uniqueness theorem for the diffeomorphism type of minimal symplectic fillings of the link of simple singularities in complex dimension 2 .

Main Theorem. Let $X$ be any minimal symplectic filling of the link of a simple singularity. Then, the diffeomorphism type of $X$ is unique. Hence, it must be diffeomorphic to the Milnor fiber. Moreover, the symplectic deformation type of $X$ is unique.

This theorem implies the following result of Brieskorn mentioned above.

Corollary. The minimal resolution of a simple singularity is diffeomorphic to the Milnor fiber of the singularity.

For other classes of isolated singularities in a complex surface, we cannot, in general, expect the uniqueness result on the diffeomorphism type of the minimal symplectic fillings of the link of the singularities. But, for the class of simple elliptic singularities, we can determine the diffeomorphism types of the minimal symplectic fillings of the link of these singularities [28]. McDuff [23] also obtained uniqueness result for another type of cyclic quotient singularities. After finishing this work, there appeared a preprint "On Lens Spaces and Their Symplectic Fillings" by P. Lisca, which announces the classification of symplectic fillings of lens spaces up to diffeomorphisms. We also noticed the following theorem as a biproduct of our uniqueness result of the symplectic filling. The proof will appear in the sequential paper [29].

Theorem. Let $M$ be a closed symplectic 4-manifold containing a pseudo-holomorphic rational curve $C$ with a $(2,3)$-cusp point. Suppose that $C$ is non-singular away from the $(2,3)$-cusp point. If the selfintersection number $C^{2}$ of $C$ is positive, then $M$ must be a rational symplectic 4-manifold and $C^{2}$ is at most 9.

\section{Preliminaries}

Firstly, we recall the notion of simple singularities and prepare some notations. Let $\Gamma \subset S U_{2}$ be a finite subgroup of $S U_{2}$. It is well known that $\Gamma$ is classified by the Dynkin diagrams of type $A_{n}(n \geq 1), D_{n}$ $(n \geq 4), E_{6}, E_{7}$ and $E_{8}$. According to these types, $\Gamma$ is isomorphic to the cyclic group $\mathbf{Z} /(n+1) \mathbf{Z}$, the binary dihedral group $\mathbf{D}_{2(n-2)}^{*}$, the binary tetrahedral group $\mathbf{T}^{*}$, the binary octahedral group $\mathbf{O}^{*}$ and 
the binary icosahedral group $\mathbf{I}^{*}$, respectively. The Dynkin diagrams of above types are diagrams with three branches of length $p, q$ and $r$. The numbers $p, q$ and $r$ are listed in Table Cartan matrix corresponding to $\Gamma$ is given by $p+q+r-2$ (see also Table $\left.\overline{1}_{1}^{\overline{1}}\right)$.

Since $\Gamma$ acts on $\mathbf{C}^{2}$ holomorphically, the quotient space $\mathbf{C}^{2} / \Gamma$ is a complex surface with a quotient singularity at the origin, which is called a simple singularity. The simple singularities can be also realized as isolated hypersurface singularities in $\mathbf{C}^{3}$ as follows. Let $f_{\Gamma}(x, y, z)$ be

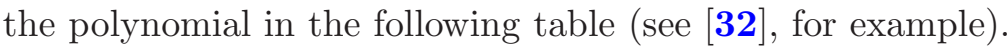

\section{Table 1.}

\begin{tabular}{|c|c|c|c|}
\hline Group $\Gamma$ & Polynomial $f_{\Gamma}(x, y, z)$ & $(p, q, r)$ & $\operatorname{rank} \Gamma$ \\
\hline$A_{n}$ & $x^{n+1}+y z$ & $(1, k, l)$ & $n$ \\
$D_{n}$ & $x^{2} y+y^{n-1}+z^{2}$ & $(2,2, n-2)$ & $n$ \\
$E_{6}$ & $x^{4}+y^{3}+z^{2}$ & $(2,3,3)$ & 6 \\
$E_{7}$ & $x^{3} y+y^{3}+z^{2}$ & $(2,3,4)$ & 7 \\
$E_{8}$ & $x^{5}+y^{3}+z^{2}$ & $(2,3,5)$ & 8 \\
\hline
\end{tabular}

Here, $(k, l)$ in the $A_{n}$-case is an arbitrary pair of positive integers satisfying $k+l=n+1$. The polynomial $f_{\Gamma}$ generates the ideal $R_{\Gamma}$ in the polynomial ring $\mathbf{C}[x, y, z]$. It turns out that $\mathbf{C}[x, y, z] / R_{\Gamma}$ is isomorphic to the ring of $\Gamma$-invariant polynomials on $\mathbf{C}^{2}$. Thus, the simple singularity of type $\Gamma$ can be realized as an isolated singularity at the origin on the surface defined by $f_{\Gamma}(x, y, z)=0$.

The link $L$ of a simple singularity is defined by

$$
L=\left\{\left.(x, y, z) \in \mathbf{C}^{3}\left|f_{\Gamma}(x, y, z)=0,\right| x\right|^{2}+|y|^{2}+|z|^{2}=\epsilon\right\},
$$

where $\epsilon$ is a small positive real number. We define the contact distribution $\xi$ on $L$ by

$$
\xi=\left\{v \in T L \mid J_{0} v \in T L\right\},
$$

where $J_{0}$ is the standard complex structure on $\mathbf{C}^{3}$. Note that $\xi$ carries a globally defined contact form $\lambda$, i.e., $\xi=\operatorname{ker} \lambda$. A compact symplectic 4-manifold $(X, \omega)$ is called a weak symplectic filling of $(L, \xi)$, if the following conditions are satisfied:

(1) $L$ is the boundary of $X$ as oriented manifolds, where $L$ is oriented by $\lambda \wedge d \lambda$ and $X$ is oriented by $\omega^{2}$,

(2) $\omega$ is positively proportional to $d \lambda$ on $\xi$.

Moreover, if there exists an extension $\tilde{\lambda}$ of $\lambda$ on a collar neighborhood of $L=\partial X$ such that $\omega=d \tilde{\lambda}$, we call $(X, \omega)$ a strong symplectic filling 
of $(L, \xi)$. If $\omega$ is exact, e.g., $L$ is a rational homology sphere, the symplectic form $\omega$ of a weak symplectic filling can be modified to another symplectic form such that $X$ becomes a strong symplectic filling (see

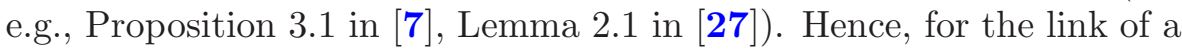
simple singularity, we may assume that our symplectic filling is a strong symplectic filling. If we replace the condition (1) above by $\left(1^{-}\right)$below, we get a notion of "(strong) concave fillings".

$\left(1^{-}\right) L$ is the boundary of $X$ and the orientations induced by the contact structure and the symplectic structure, respectively, are opposite.

If $(X, \omega)$ is a strong symplectic filling of $(L, \xi)$, a collar neighborhood of $\partial X$ can be embedded in the symplectization of $(L, \xi)$ such that the outward normal vector of $X$ points to the positive side. For strong concave fillings, we have a similar embedding, but the outward normal vector points to the negative side. Hence, we can glue a strong symplectic filling $X$ and a strong concave filling $Y$ to get a closed symplectic manifold possibly after modifying the symplectic structures.

\section{Outline of the proof of Main Theorem}

In this section, we give the outline of our argument and the organization of this paper. Roughly speaking, the proof of Main Theorem consists of two parts.

The first part is that the symplectic filling $X$ is embedded into a certain smooth closed symplectic 4-manifold $Z(\Gamma)$, which will be called a compactification of $X$. This is similar to the one in [27], but we investigate the situation more precisely. Namely, we will determine $Z(\Gamma)$ and the compactifying divisor explicitly in Section $\overline{3}$. Let $M_{\Gamma}$ be the Milnor fiber defined by $\left\{(x, y, z) \in \mathbf{C}^{3} \mid f_{\Gamma}(x, y, z)^{-}=1\right\}$, where $f_{\Gamma}(x, y, z)$ is the polynomial in Table ${ }_{1}^{1}$. We regard $f_{\Gamma}(x, y, z)$ as a weighted homogeneous polynomial. The weights and degrees are given by the following table.

\section{Table 2.}

\begin{tabular}{|c|c|c|}
\hline Group $\Gamma$ & Weights $(a, b, c)$ & degree $h$ \\
\hline$A_{n}$ & $(1, k, l)$ & $n+1$ \\
$D_{n}$ & $(n-2,2, n-1)$ & $2(n-1)$ \\
$E_{6}$ & $(3,4,6)$ & 12 \\
$E_{7}$ & $(4,6,9)$ & 18 \\
$E_{8}$ & $(6,10,15)$ & 30 \\
\hline
\end{tabular}

We recall a compactification of the Milnor fiber of a simple singularity after Saito. For more information, see [31]. Consider the 
weighted projective space $\mathbf{P}(a, b, c, 1)=\left(\mathbf{C}^{4} \backslash 0\right) / \sim$, where $(x, y, z, w) \sim$ $\left(t^{a} x, t^{b} y, t^{c} z, t w\right)$ for $t \in \mathbf{C}^{*}$. By a map $\iota$ from $\mathbf{C}^{3}$ to $\mathbf{P}(a, b, c, 1)$ defined by $\iota(x, y, z)=[x, y, z, 1], \mathbf{C}^{3}$ can be embedded as an open dense subset so that $\mathbf{P}(a, b, c, 1)=\mathbf{C}^{3} \cup \mathbf{P}(a, b, c)$. Here, $\mathbf{P}(a, b, c)=\{[x, y, z, 0] \in$ $\mathbf{P}(a, b, c, 1)\}$. Denote by $\overline{M_{\Gamma}}$ the closure of $\iota\left(M_{\Gamma}\right)$ in $\mathbf{P}(a, b, c, 1)$, which is the hypersurface defined by $f_{\Gamma}(x, y, z)=w^{h}$ in $\mathbf{P}(a, b, c, 1)$. It is singular, when $\Gamma$ is not of the $A_{1}$-type. The singular points of $\overline{M_{\Gamma}}$ lie on $C=\overline{M_{\Gamma}} \backslash \iota\left(M_{\Gamma}\right)=\overline{M_{\Gamma}} \cap \mathbf{P}(a, b, c)$, which is a smooth rational curve. These singularities turn out to be cyclic quotient singularities of type $A_{n, 1}$, where $n=p, q, r$. Taking the minimal resolution $\pi: \widetilde{M_{\Gamma}} \rightarrow \overline{M_{\Gamma}}$, we have a compact projective smooth surface $\widetilde{M_{\Gamma}}$, which contains the Milnor fiber $M_{\Gamma}$ as an open dense subset.

A little modification is necessary for $A_{n}$-singularities. The equations of type $A_{n}$ in Table same argument works. The reason why we use $x^{n+1}+y z$ is two-fold. Firstly, we discuss cases both for $n$ even and odd, while the cases with even $n$ are not discussed in [31]. Secondly, it seems more convenient in the argument in Section 3 with this equation and we choose the weights $(1, k, l)=(1,2, n-1)$ when $n \geq 2$. Anyway, in the $A_{n}$-case, some of $p, q, r$ in Table $\overline{1}$ are 1 and the number of singularities on $\overline{M_{\Gamma}}$ is less than 3 . To deal with all the cases systematically, in the $A_{n}$-case, we also blow-up at an appropriate number of non-singular points on $C$ and denote the result by $\widetilde{M_{\Gamma}}$. This gives the following configuration of the compactifying divisor for any $\Gamma$.

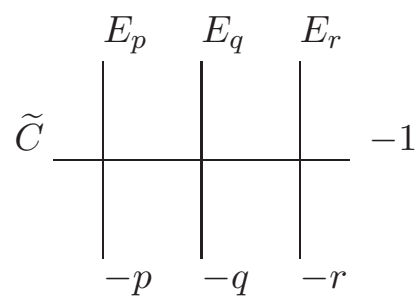

Figure 1.

We denote by $\widetilde{C}$ the proper transform of $C$, which has the selfintersection number -1 and by $E_{p}, E_{q}$ and $E_{r}$ the exceptional set for the resolution or blow-up with the self-intersection number $E_{n}^{2}=-n$ for $n=p, q, r$ respectively. Here, the integers $p, q$ and $r$ are given by Table $\bar{I}_{1}$. Each of the curves $E_{p}, E_{q}$ and $E_{r}$ intersects with $\widetilde{C}$ at one point transversely. Saito also showed that 


$$
K_{\widetilde{M_{\Gamma}}}=-\left(2 \widetilde{C}+E_{p}+E_{q}+E_{r}\right) .
$$

In particular, $-K_{\widetilde{M_{\Gamma}}}$ is an effective divisor. Write

$$
M_{\Gamma}^{\text {out }}=\widetilde{M_{\Gamma}} \backslash \iota\left(M_{\Gamma} \cap B^{6}\right),
$$

which is a regular neighborhood of $\widetilde{C} \cup E_{p} \cup E_{q} \cup E_{r}$. Here, $B^{6}$ is the closed unit disc around the origin in $\mathbf{C}^{3}$. Note that $M_{\Gamma}^{\text {out }}$ is a strong concave filling in the sense of Section $X$ and $M_{\Gamma}^{\text {out }}$ to obtain a closed symplectic manifold $(Z(\Gamma), \omega)$ :

$$
Z(\Gamma):=X \cup_{S^{3} / \Gamma} M_{\Gamma}^{\text {out }}
$$

which we call a compactification of the filling $X$. We apply the following theorem in [26] to $Z(\Gamma)$, which was independently proved in [21], see

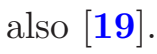

Theorem 2.3. Let $(M, \omega)$ be a closed symplectic 4-manifold such that

$$
\int_{M} c_{1}(M) \wedge \omega>0
$$

Then, $M$ is a rational or ruled symplectic 4-manifold up to blow-up and down.

We briefly recall the argument in [26]. Using Taubes' result, we find a symplectically embedded 2 -sphere with non-negative self-intersection number in $(M, \omega)$. Then, a theorem by McDuff [23] states that $(M, \omega)$ must be a rational or ruled symplectic 4-manifold, the symplectic deformation type of which is also uniquely determined. (As for classifi-

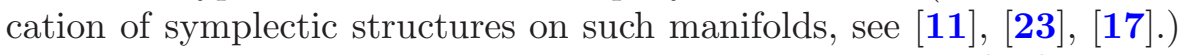
Namely, after blowing-down a family of disjoint symplectic $(-1)$-curves, we get a minimal symplectic 4-manifold, which is symplectic deformation equivalent either to the complex projective plane or to a minimal ruled symplectic 4-manifold. In fact, we will describe, in Section 3, the diffeomorphism type of $Z(\Gamma)$ explicitly (Theorem $3.1-1)$. The symplectic filling $X$ is the complement of a regular neighborhood of the compactifying divisor, as in Figure in in $Z(\Gamma)$.

The second part of the proof of Main Theorem is to determine how the compactifying divisor $\widetilde{C} \cup E_{p} \cup E_{q} \cup E_{r}$ is embedded in $Z(\Gamma)$. We will show that the complement of the compactifying divisor is diffeomorphic to a certain standard one which corresponds to the case when $X$ is the Milnor fiber. Here, we give an outline of our argument in the case of $E_{n}$. Some modification is needed in the cases of $A_{n}$ and $D_{n}$ (see Sections $\overline{4} \cdot \overline{2}$ and 5.2. After preparing some fundamental lemmas in Section $\overline{4} .1$, we make 
blow-up and down operations on $Z(\Gamma)$ and reduce the problem to the one concerning the image $D$ of the compactifying divisor in $\mathbf{C} P^{2}$. We will see that $D$ is a pseudo-holomorphic curve, of degree 3 , with one $(2,3)$ cusp singularity in $\mathbf{C} P^{2}$ with respect to a certain tame almost complex structure (Section $4 . \overline{2}$ ), which we shall call a pseudo-holomorphic cuspidal cubic curve. Here, a singular point of a pseudo-holomorphic curve is called a $(2,3)$-cusp point if its parametrization around the singular point has the form $\left(z^{2}, z^{3}\right)+O(4)$ (see [24]). Similarly, a pseudo-holomorphic curve in $\mathbf{C} P^{1} \times \mathbf{C} P^{1}$ is called a pseudo-holomorphic cuspidal curve of bidegree $(2,2)$, if it is a pseudo-holomorphic curve with one $(2,3)$-cusp singularity, which represents the class [2,2], i.e., $2\left[\mathbf{C} P^{1} \times\{p t\}\right]+2\left[\{p t\} \times \mathbf{C} P^{1}\right]$. By the adjunction formula, a pseudoholomorphic rational curve representing the class $3\left[\mathbf{C} P^{1}\right]$ (resp. $\left.[2,2]\right)$ in $\mathbf{C} P^{2}$ (resp. $\left.\mathbf{C} P^{1} \times \mathbf{C} P^{1}\right)$ must have exactly one singular point, which is either a node or a non-immersed point of multiplicity 2. In Sections and $\overline{5} . \mathbf{2}$, we always assume that the singular point is fixed at $p$ and the almost complex structure around $p$ is integrable. So, the Taylor expansion above is the usual description of a holomorphic map germ.

Then, we will show a uniqueness result for the diffeomorphism type of the complements $\mathbf{C} P^{2} \backslash D$ (Section, isotopy problem for singular pseudo-holomorphic curves. In general, the uniqueness of symplectic isotopy types of singular pseudo-holomorphic curves does not hold. For example, it is classically known that there exist pairs of algebraic curves of degree 6 with 6 cusp points in $\mathbf{C} P^{2}$ which

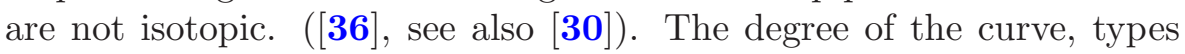
of the singular points and the number of the singular points strongly affect the nature of this problem. In this paper, we will achieve uniqueness for pseudo-holomorphic cuspidal cubic curves in $\mathbf{C} P^{2}$. Namely, the degree of our curve is 3 and the number of cusp point is 1 . The $(2,3)$-cusp singularity has a nice special feature as follows. Although a map germ $(\mathbf{C}, O) \rightarrow\left(\mathbf{C}^{2}, O\right)$ may be perturbed to another map germ, which is not equivalent to the initial one in general, by adding higher order terms $[\mathbf{\overline { \mathbf { 6 } }}]$, the map germ of $f(z)=\left(z^{2}, z^{3}\right)+O(4)$ is equivalent to $f_{0}(z)=\left(z^{2}, z^{3}\right)$. This point will be discussed in Section Theorem 5. proved in Section 5.1 is another important ingredient to prove the uniqueness. This is derived from a cobordism argument which is related to transversality and compactness properties. Here, the conditions on the degree and the number of cusp points are essentially used. Since this kind of statement does not hold for Zariski's example as we mentioned above, we cannot expect the uniqueness in that case. 
Combining the description of the symplectic filling $X$ as a complement of the compactifying divisor in $Z(\Gamma)$ (Section 4) and the uniqueness result in Section 5, we will complete the proof of Main theorem in Section $\overline{6}$.

\section{Description of the compactification $Z(\Gamma)$}

In this section, we show the following:

Theorem 3.1. The compactification $Z(\Gamma)$ is a rational or ruled symplectic 4-manifold up to blow up and down. More explicitly, $Z(\Gamma)$ is symplectomorphic to an $n+3$-point blow-up of the complex projective plane with an appropriate Kähler form. In particular, it is diffeomorphic to the following rational symplectic 4-manifold in Table $\mathbf{B}_{-1}^{-}$according to the type of $\Gamma$.

Table 3.

\begin{tabular}{|c|c|}
\hline Type of $\Gamma$ & Diffeomorphism type of $Z(\Gamma)$ \\
\hline$A_{n}$ & $\mathbf{C} P^{2} \#(3+n) \overline{\mathbf{C} P^{2}}$ \\
$D_{n}$ & $\mathbf{C} P^{2} \#(3+n) \overline{\mathbf{C} P^{2}}$ \\
$E_{6}$ & $\mathbf{C} P^{2} \# 9 \overline{\mathbf{C} P^{2}}$ \\
$E_{7}$ & $\mathbf{C} P^{2} \# 10 \overline{\mathbf{C} P^{2}}$ \\
$E_{8}$ & $\mathbf{C} P^{2} \# 11 \overline{\mathbf{C} P^{2}}$ \\
\hline
\end{tabular}

Proof. To prove the first part of Theorem $\overline{3} \cdot 1$, it suffices to show that $Z(\Gamma)$ satisfies the assumption in Theorem 2.3. Recall that $2 \widetilde{C}+E_{p}+$ $E_{q}+E_{r}$ is an anti-canonical divisor of $\widetilde{M_{\Gamma}}$, which is contained in $M_{\Gamma}^{\text {out }}$.

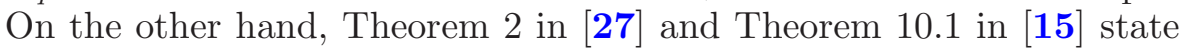
that the canonical bundle $K_{X}$ of any minimal symplectic filling $X$ of $S^{3} / \Gamma$ with the quotient contact structure is trivial. Since $S^{3} / \Gamma$ is a rational homology 3 -sphere, we conclude that $2 \widetilde{C}+E_{p}+E_{q}+E_{r}$ is also an anti-canonical divisor of $Z(\Gamma)$. Note that it is written as a positive combination of symplectically embedded surfaces. Hence, we have

$$
\int_{Z(\Gamma)} c_{1}(T Z(\Gamma)) \wedge \omega=2 \int_{\widetilde{C}} \omega+\int_{E_{p}} \omega+\int_{E_{q}} \omega+\int_{E_{r}} \omega>0 .
$$

This is the inequality in the assumption of Theorem

Next, we shall prove the second part. To do this, we need more detailed information on the topology of the symplectic filling $X$. The following proposition is an improvement of a result in [27]. 
Proposition 3.2. Let $X$ be any minimal symplectic filling of the link of the simple singularity of type $\Gamma$. Then, the intersection form of $X$ is negative definite and $b_{2}^{-}(X)=\operatorname{rank} \Gamma$. Here, $\operatorname{rank} \Gamma$ is the rank of the Cartan matrix corresponding to the Dynkin diagram of $\Gamma$.

Remark 3.3. The fact that $X$ is negative definite is the conclusion

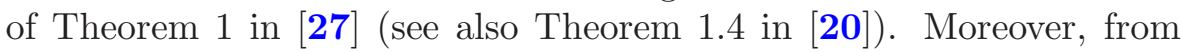
Theorem 2 in [27] and Theorem 10.1 in [1; bundle $K_{X}$ is always trivial. In particular, $X$ is a spin manifold. Then, using Froyshov's result [8], we obtain the following estimate

$$
b_{2}^{-}(X) \leq \operatorname{rank} \Gamma .
$$

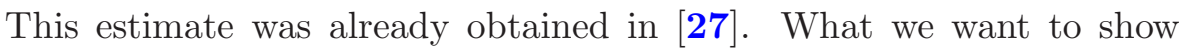
here is that the equality exactly holds in (1). We would also like to mention that we do not use Froyshov's result to prove it in this paper.

We prepare a couple of lemmas.

Lemma 3.5. We have $b_{1}\left(M_{\Gamma}^{\text {out }}\right)=0$.

Proof. Note that $M_{\Gamma}^{\text {out }}$ is a regular neighborhood of the configuration of rational curves as in Figure $]_{1}^{1}$. Then, the conclusion is obvious. q.e.d.

Next, we prove the following:

Lemma 3.6. For any symplectic filling $X$ of the link of the simple singularity of type $\Gamma$, we have $b_{1}(X)=0$.

Proof. Suppose that $b_{1}(X) \neq 0$, then we have a non-trivial abelian covering $p: \widetilde{X} \rightarrow X$ of any degree $d>1$. Let us take $d$ bigger than the order of $\Gamma$, which is certainly isomorphic to the fundamental group of $\partial X$. Then, the boundary $\partial \widetilde{X}$ consists of at least two connected components, each of which is isomorphic to $S^{3} / \Gamma_{i}^{\prime}$ for some finite subgroup $\Gamma^{\prime} \subset S U_{2}$. By pulling back the symplectic from $\omega$ on $X$ to $\widetilde{X}$ by $p, \widetilde{X}$ is also a symplectic 4-manifold with disconnected boundary $\bigsqcup_{i} S^{3} / \Gamma_{i}^{\prime}$.

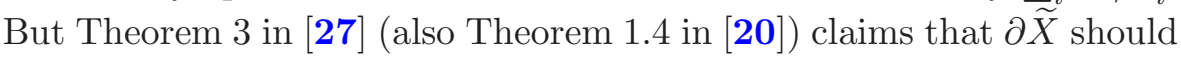
be connected, which is a contradiction. $\quad$ q.e.d.

Now, we show the following:

Lemma 3.7. For the closed symplectic 4-manifold $Z(\Gamma)$ in (12.2. have $b_{1}(Z(\Gamma))=0$.

Proof. We apply the Mayer-Vietoris exact sequence (over Q-coefficients) to the decomposition in $\left(\overline{2} \cdot 2_{1}^{1}\right)$. Then, by Lemmas $3 . \overline{3}$ and imply Lemma 
Proof of Proposition $=0$ for the minimal symplectic filling $X$, we have

$$
c_{1}^{2}\left(K_{Z(\Gamma)}\right)=c_{1}^{2}\left(K_{M_{\Gamma}^{\text {out }}}\right)=c_{1}^{2}\left(K_{\widetilde{M_{\Gamma}}}\right)=8-(p+q+r) .
$$

On the other hand, we have $b_{2}(Z(\Gamma))=b_{2}(X)+4$, and $\sigma(Z(\Gamma))=$ $\sigma(X)+\sigma\left(M_{\Gamma}^{\text {out }}\right)=\sigma(X)-2$ (Novikov additivity for signatures). It follows that

$$
2 \chi(Z(\Gamma))+3 \sigma(Z(\Gamma))=6-4 b_{1}(Z(\Gamma))+5 b_{2}^{+}(X)-b_{2}^{-}(X) .
$$

Since $b_{2}^{+}(X)=0$ and $b_{1}(Z(\Gamma))=0$ by Lemma 3. lowing equality

$$
2 \chi(Z(\Gamma))+3 \sigma(Z(\Gamma))=6-b_{2}^{-}(X) .
$$

Note that $c_{1}^{2}\left(K_{Z(\Gamma)}\right)=2 \chi(Z(\Gamma))+3 \sigma(Z(\Gamma))$. Combining these equalities, $b_{2}^{-}(X)$ can be calculated as

$$
b_{2}^{-}(X)=p+q+r-2 .
$$

Hence, we obtain Proposition

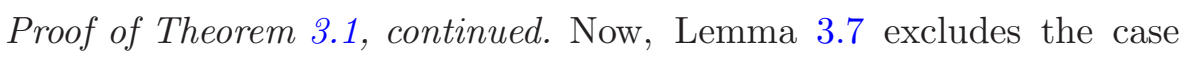
that $Z(\Gamma)$ is diffeomorphic to any ruled surface over a Riemann surface of genus $\geq 1$. There are two symplectic deformation types of minimal rational or rationally ruled symplectic 4-manifolds, i.e., $\mathbf{C} P^{2}$ and $\mathbf{C} P^{1} \times \mathbf{C} P^{1}$. Note that the one point blow-up of $\mathbf{C} P^{1} \times \mathbf{C} P^{1}$ has the same symplectic deformation type as the two point blow-up of $\mathbf{C} P^{2}$. Furthermore, we have $b_{2}^{+}(Z(\Gamma))=b_{2}^{+}\left(M_{\Gamma}^{\text {out }}\right)=1$ and

$$
b_{2}^{-}(Z(\Gamma))=b_{2}^{-}(X)+b_{2}^{-}\left(M_{\Gamma}^{\text {out }}\right)=p+q+r+1 .
$$

Hence, we can determine the diffeomorphism types of $Z(\Gamma)$, which completes the proof of Theorem 3.11 .

q.e.d.

\section{Contraction of exceptional rational curves}

In this section, we make blow-up and down operations on the symplectic 4-manifold $Z(\Gamma)$ constructed in section 3 to transform the compactifying divisor $\widetilde{C} \cup E_{p} \cup E_{q} \cup E_{r}$ to a pseudo holomorphic singular curve in a rational symplectic 4-manifold. We shall describe the singular curve explicitly. 
4.1. Some lemmas. First of all, we prove a fundamental lemma about pseudo holomorphicity of the image of a pseudo holomorphic curve by the contraction of pseudo holomorphic $(-1)$-curves. In section $\overline{4} . \overline{2}$, we frequently use Lemma is combined with Lemma is based on Lemma 3.2 in [23]. Take and fix a tame almost complex structure $J$ on a symplectic 4 -manifold $(M, \omega)$ and $E$ a $J$-holomorphic $(-1)$-curve. We denote by $\pi: M \rightarrow \bar{M}$ the contraction of $E$ in $C^{\infty}$ category. Note that $\bar{M}$ is diffeomorphic to the symplectic blown down manifold along $E$. Denote by $\bar{\omega}$ the symplectic form on $\bar{M}$ as in Lemma 3.2 in [23].

Lemma 4.1. Let $S$ be a J-holomorphic curve in $(M, \omega)$, which intersects $E$ transversely at non-singular points on $S$. Then, there exists a tame almost complex structure $\bar{J}$ on the blown-down manifold $(\bar{M}, \bar{\omega})$ so that $\pi(S)$ is $\bar{J}$-holomorphic.

Proof. Firstly, we take a tame almost complex structure $J^{\prime}$ on $(M, \omega)$ satisfying the following properties:

(4.2.1) $J^{\prime}$ is integrable in a neighborhood $U$ of $E$.

(4.2.2) $J^{\prime}$ coincides with $J$ along $E$, i.e., $\left.J^{\prime}\right|_{E}=\left.J\right|_{E}:\left.\left.T M\right|_{E} \rightarrow T M\right|_{E}$.

(4.2.3) $J^{\prime}$ coincides with $J$ outside of a neighborhood $V$ of $E$, where $\bar{U} \subset$ $V$.

(4.2.4) Each component of $S \cap U$ is $J^{\prime}$-holomorphic.

In fact, such a $J^{\prime}$ can be found as follows. Pick a $J$-invariant subbundle $N$, which is complementary to $T E$ in $\left.T M\right|_{E}$. Obviously, $N$ is a complex line bundle. Pick a hermitian connection $\nabla$ on $N$. Since $E$ is a Riemann surface, $N$ becomes a holomorphic line bundle by $\bar{\partial}=\nabla^{0,1}$. With respect to this complex structure, $N$ is a complex manifold and $E$ is a complex submanifold. Denote by $V$ a suitable tubular neighborhood of $E$ in $M$ and by $D(N)$ a disk bundle in $N$. Here, we take $V$ so that $V \cap S$ is in the smooth part of $S$. Pick a diffeomorphism $\phi: D(N) \rightarrow V$ in such a way that the image of each fiber is $J$-holomorphic at the origin in the fiber, and $\left.\phi\right|_{E}$ is the identity. Hence, we get an integrable complex structure $J_{0}$ on $V$ so that $J_{0}$ coincides with $J$ along $E$. Note that $S$ is $J$-holomorphic, hence the tangent spaces of $S$ at intersection points of $S$ and $E$ are $J_{0}$-holomorphic. Since $S$ intersects $E$ transversely, we can assume that $\phi$ maps the union of some fibers onto $S \cap V$.

Clearly, $J_{0}$ is $\omega$-tame on a small neighborhood $\bar{U} \subset V$ of $E$. Then, we can deform $J_{0}$ and $J$ on $V \backslash U$ through $\omega$-tame almost complex structures to get a desired $J^{\prime}$ on $M$. Moreover, we can choose the deformation $J_{t}$ from $J_{0}$ to $J$ on $V \backslash U$ so that $S \cap(V \backslash U)$ is pseudo-holomorphic with 
respect to each $J_{t}$, since $S \cap(V \backslash U)$ is a properly embedded pseudoholomorphic curve with respect to $J_{0}$ and $J$. In particular, $S$ is $J^{\prime}$ holomorphic.

Now, we are going to prove Lemma $\overline{4} .1$. Denote by $\pi: M \rightarrow \bar{M}$ the contraction of $E$. Lemma 3.2 in [203] assures existence of a symplectic structure $\bar{\omega}$ on $\bar{M}$ so that $\bar{\omega}$ coincides $\pi^{*} \omega$ outside of $U$ and an $\bar{\omega}$-tame almost complex structure $\bar{J}$ on $\bar{M}$ such that $\bar{J} \circ \pi_{*}=\pi_{*} \circ J^{\prime}$.

Since $S$ is $J^{\prime}$-holomorphic, hence $\pi(S)$ is $\bar{J}$-holomorphic. $\quad$ q.e.d.

There are several choices in the proof of Lemma $\overline{4} .1$, e.g., choices of $N$, the hermitian metric and the hermitian connection on $N$, and $J^{\prime}$ in (4.2). However, we remark that all of them are chosen in contractible families. Therefore, we also get the following:

Lemma 4.3. Let $J_{t}$ be a family of tame almost complex structures on $(M, \omega)$ and $E_{t}$ a family of $J_{t}$-holomorphic $(-1)$-curves. Denote by $\pi_{t}: M \rightarrow \bar{M}$ the contraction of $E_{t}$. Let $S_{t}$ be a family of $J_{t}$-holomorphic curves in $(M, \omega)$, which intersects $E_{t}$ transversely at non-singular points on $S_{t}$. Then, there exists a family of tame almost complex structures $\overline{J_{t}}$ on $\left(\bar{M}, \overline{\omega_{t}}\right)$ so that $\pi_{t}\left(S_{t}\right)$ is $\overline{J_{t}}$-holomorphic for each $t$.

We call a 2-dimensional homology class $e$ a symplectic (-1)-class, if $e$ contains a symplectic ( -1$)$-curve, i.e., a symplectically embedded sphere of self-intersection number -1 .

We show the following useful lemma.

Lemma 4.4. Let $D$ be an irreducible J-holomorphic curve in a symplectic 4-manifold $M$. Suppose that $D$ represents the anti-canonical class, i.e., the homology class $[D]$ is the Poincaré dual of the first Chern class $c_{1}(M)$. Then, there exists a tame almost complex structure $J^{\prime}$ such that $J^{\prime}$ coincides with $J$ in a neighborhood of $D$ and any symplectic $(-1)$-class $e$ is represented by a unique $J^{\prime}$-holomorphic embedded representative $E$. Moreover, $E$ intersects $D$ at exactly one regular point transversely.

Remark 4.5. It is shown in [23] that any symplectic (-1)-class is represented by a $J$-holomorphic embedded sphere for a generic tame almost complex structure $J$. In Lemma 4.4 , the almost complex structure $J^{\prime}$ is not generic in the whole space of tame almost complex structures, since $J^{\prime}$ is prescribed in a neighborhood of $D$. Since we only need properties (1) and (2) in the proof of Lemma 4.4, , we can find $J^{\prime}$ arbitrary close to $J$.

Proof. We pick a tame almost complex structure $J^{\prime}$ satisfying the following conditions: 
(1) $J^{\prime}$ coincides with $J$ in a neighborhood of $D$, in particular, $D$ is $J^{\prime}$-holomorphic,

(2) there are no $J^{\prime}$-holomorphic spheres with self-intersection number less than -1 , except possibly $D$, in particular, there are no embedded $J^{\prime}$-holomorphic spheres with self-intersection number less than -1 .

This is possible, since the virtual dimension of the moduli space of pseudo-holomorphic curves as in (2) is negative.

Note that the class $e$ has a unique pseudo-holomorphic representative for a generic tame almost complex structure, which is necessarily embedded. We pick a sequence $\left\{J_{n}\right\}$ of generic tame almost complex structures converging to $J^{\prime}$. Denote by $E_{n}$ the $J_{n}$-holomorphic representative of the class $e$.

Suppose that $E_{n}$ converges to some $J^{\prime}$-holomorphic sphere $E$. Since the self-intersection number of $e$ is $-1, E$ is not multiply covered. Then, the fact that the virtual genus of the class $e$ is 0 implies that $E$ is an embedded $J^{\prime}$-holomorphic sphere representing the class $e$, which is the desired $J^{\prime}$-holomorphic $(-1)$-curve. (Here, we call the number $(e \cdot e-$ $\left.c_{1}(M) \cdot e+2\right) / 2$ the virtual genus of the class $e$.)

Suppose that $E_{n}$ converges as stable maps to $f: \cup \mathbf{C} P_{(i)}^{1} \rightarrow M$ consisting of at least two components and write $f\left(\mathbf{C} P_{(i)}^{1}\right)=B_{i}$. We may assume that at least one of $B_{i}$ is a multiple of $D$ (possibly $D$ itself).

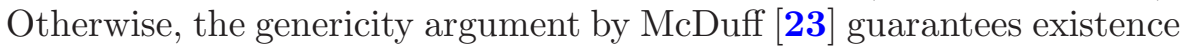
of $J^{\prime}$-holomorphically embedded 2 -sphere representing the class $e$. We denote by $k[D]$ the sum of all homology classes $\left[B_{i}\right]$, which are multiples of $[D]$. For other $B_{i}$, we write $B_{i}=l_{i} C_{i}$ with $l_{i} \geq 1$ and $C_{i}$ being the images of simple (i.e., not multiply covered) $J^{\prime}$-holomorphic maps. Since no $C_{i}$ is contained in a neighborhood of $D$, the genericity of $J^{\prime}$ assures transversality for somewhere injective $J^{\prime}$-holomorphic spheres $C_{i}$. Hence, for a sufficiently large $n$, the homology classes $\left\{C_{i}\right\}$ are represented by $J_{n}$-holomorphic spheres $C_{i, n}$. This implies that the intersection numbers $C_{i} \cdot e=C_{i, n} \cdot E_{n}$ are non-negative:

$$
C_{i} \cdot\left(\sum l_{i} C_{i}+k D\right) \geq 0
$$

On the other hand, $e$ is a symplectic $(-1)$-class, hence, we have $c_{1}(M) e=$ 1. Since $D$ represents the anti-canonical class of $M$, we find

$$
D \cdot\left(\sum l_{i} C_{i}+k D\right)=1 \text {. }
$$

Since $k$ and $l_{i}$ are positive integers, these two inequalities imply that

$$
e^{2}=\left(\sum l_{i} C_{i}+k D\right)^{2}>0
$$


which contradicts to $e^{2}=-1$.

Let $E$ be the $J^{\prime}$-holomorphic $(-1)$-sphere representing the class $e$. Since $D \cdot E=1$ and $E$ and $D$ are $J^{\prime}$-holomorphic curves, $E$ and $D$ intersects exactly at one point transversely.

q.e.d.

Remark 4.6. Under the assumption of Lemma $4 . \overline{4}$, the image $\bar{D}$ of $D$ by the blow-down is an anti-canonical divisor of the blow-down manifold. Pseudo-holomorphicity of $\bar{D}$ is guaranteed by Lemma $\overline{4} . \overline{1}$.

4.2. Contraction of exceptional curves in $Z(\Gamma)$. Throughout this Section 4.2 , we pick a tame almost complex structure on $Z(\Gamma)$, which is integrable near the compactifying divisor.

Case: $E_{n}$ with $(p, q, r)=(2,3, n-3), n=6,7,8$.

We recall that we have the $(-1)$-curve $\widetilde{C}$ in $M_{E_{n}}^{\text {out }} \subset Z\left(E_{n}\right)$. We can blow down $Z\left(E_{n}\right)$ along $\widetilde{C}$ to get the 4-manifold $Z\left(E_{n}\right)_{(1)}$. Then, the curve $E_{p}$ becomes the $(-1)$-curve $E_{p}^{\prime}$. We contract the $(-1)$-curve $E_{p}^{\prime}$ to get the 4-manifold $Z\left(E_{n}\right)_{(2)}$. Then, we can see that the $(-1)$-curve $E_{q}^{\prime \prime}$ and the $(-n+5)$-curve $E_{r}^{\prime \prime}$, (note that $\left.-r+2=-n+5\right)$, intersect as in the following picture.

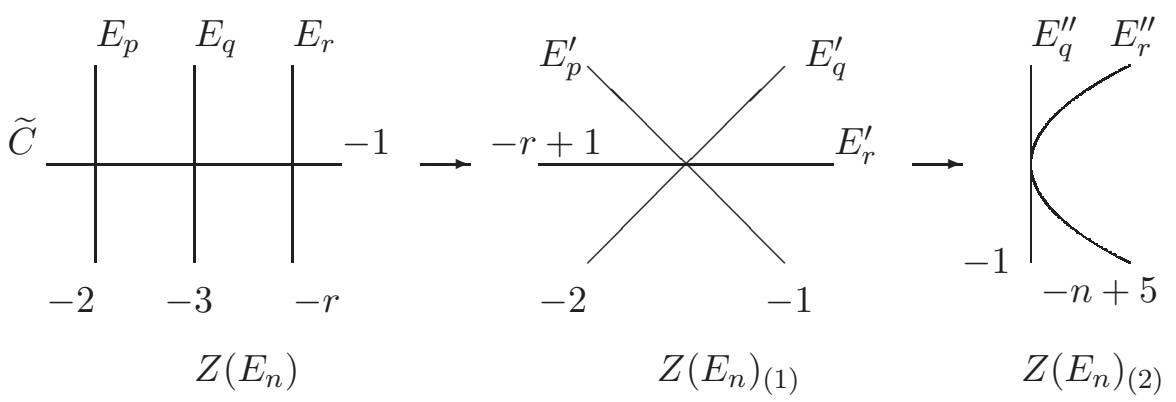

Figure 2.

Furthermore, we contract $E_{q}^{\prime \prime}$ to get the 4-manifold $Z\left(E_{n}\right)_{(3)}$ which contains the singular curve $E_{r}^{\prime \prime \prime}$. Its self-intersection number is $-n+9$. From Theorem 1,1, it is easy to see that $Z\left(E_{n}\right)_{(3)}$ is symplectic deformation equivalent to $\mathbf{C} P^{2} \# n \overline{\mathbf{C} P^{2}}$. Thus, we can blow down $Z\left(E_{n}\right)_{(3)}$ along further $n(-1)$-curves to get $\mathbf{C} P^{2}$. It is, however, necessary to know how $E_{r}^{\prime \prime \prime}$ is transformed under the blow-down process. For this purpose, we use Lemma $\overline{4}$. to make exceptional curves of the blowdown process and $E_{r}^{\prime \prime \prime}$ pseudo-holomorphic, with respect to a common tame almost complex structure, simultaneously. Note that $E_{r}^{\prime \prime \prime}$ represents the anti-canonical class. Up to this stage $Z\left(E_{n}\right)_{(3)}$, the blow-down 
is carried out also as complex blow-down and the complex structure near the compactifying divisor descends to the one in a neighborhood of the $(2,3)$-cusp point of $E_{r}^{\prime \prime \prime}$ in $Z\left(E_{n}\right)_{(3)}$. Hence, we may assume that the almost complex structure is integrable in a neighborhood of $E_{r}^{\prime \prime \prime}$, the effect to $E_{r}^{\prime \prime \prime}$ under these blow-down is the same as in complex geometry. Note also that McDuff showed that any closed symplectic 4-manifold becomes minimal by blow-down a maximal family of disjoint symplectic $(-1)$-curves simultaneously [23i].

More precisely, we show the following:

Proposition 4.7. Let $\epsilon_{j}, j=1, \ldots, n$, be a maximal family of disjoint $(-1)$-rational curves in $Z\left(E_{n}\right)_{(3)}$, which is diffeomorphic to $\mathbf{C} P^{2} \# n \overline{\mathbf{C} P^{2}}$. Let $\pi: Z\left(E_{n}\right)_{(3)} \rightarrow \mathbf{C} P^{2}$ be a smooth mapping corresponding to the blowing down along the $\epsilon_{j}$ 's. Then, $\pi\left(E_{r}^{\prime \prime \prime}\right)$ is represented by a pseudo holomorphic cuspidal cubic curve.

Proof. Choose a maximal family of disjoint symplectic $(-1)$-curves in $Z(\Gamma)$ to get $\mathbf{C} P^{2}$. Applying Lemma with $D=E_{r}^{\prime \prime \prime}$, then we may assume that $\epsilon_{j}$ and $E_{r}^{\prime \prime \prime}$ are pseudo-holomorphic with respect to a common almost complex structure. Moreover, $\epsilon_{j}$ and $E_{r}^{\prime \prime \prime}$ intersect only at one point and transversely. Therefore, the only singularity of $\pi\left(E_{r}^{\prime \prime \prime}\right)$ is the image of the one in $E_{r}^{\prime \prime \prime}$. Since we choose our almost complex structure integrable near this curve, the type of the singularity is the same as in complex geometry, i.e., a $(2,3)$-cusp. We also find that the self-intersection number of $\pi\left(E_{r}^{\prime \prime \prime}\right)$ must be $(-n+9)+n=9$, which implies that its homology class is $3\left[\mathbf{C} P^{1}\right] \in \mathrm{H}_{2}\left(\mathbf{C} P^{2}\right)$. Thus, we get the conclusion.

q.e.d.

Case: $D_{n}$ with $(p, q, r)=(2,2, n-2)$.

We recall the compactification in section 2 (also Figure $\overline{1}(\overline{1})$ and blowdown $(-1)$-curves in it. We first contract the central $(-1)$-curve $\widetilde{C}$ and then contract the image $E_{q}^{\prime}$ of $E_{q}$ (see the first two steps in Figure isis). We denote the blown down manifold at this stage by $Z\left(D_{n}\right)_{(2)}$. (One rational curve $E_{p}^{\prime \prime}$ with self-intersection number 0 and the other rational curve $E_{r}^{\prime \prime}$ with self-intersection number $-n+4$, which are tangents to each other).

We blow up at a point on $E_{p}^{\prime \prime}$, which becomes a $(-1)$-curve $\widetilde{E}_{p}^{\prime \prime}$. We contract $\widetilde{E}_{p}^{\prime \prime}$ next (see the last three steps in Figure ${ }_{-1}^{13}$ ). We denote the manifold at this stage by $Z\left(D_{n}\right)_{(3)}^{(1)}$. The compactifying divisor $\widetilde{C} \cup E_{p} \cup$ $E_{q} \cup E_{r}$ is transformed to $A \cup D$, where $A=e_{1}^{\prime}$ is a rational curve of self-intersection number 0 and $D=E_{r}^{\prime \prime \prime}$ is a cuspidal rational curve of self-intersection number $-n+8$. They intersect at the cusp point of $D$. 


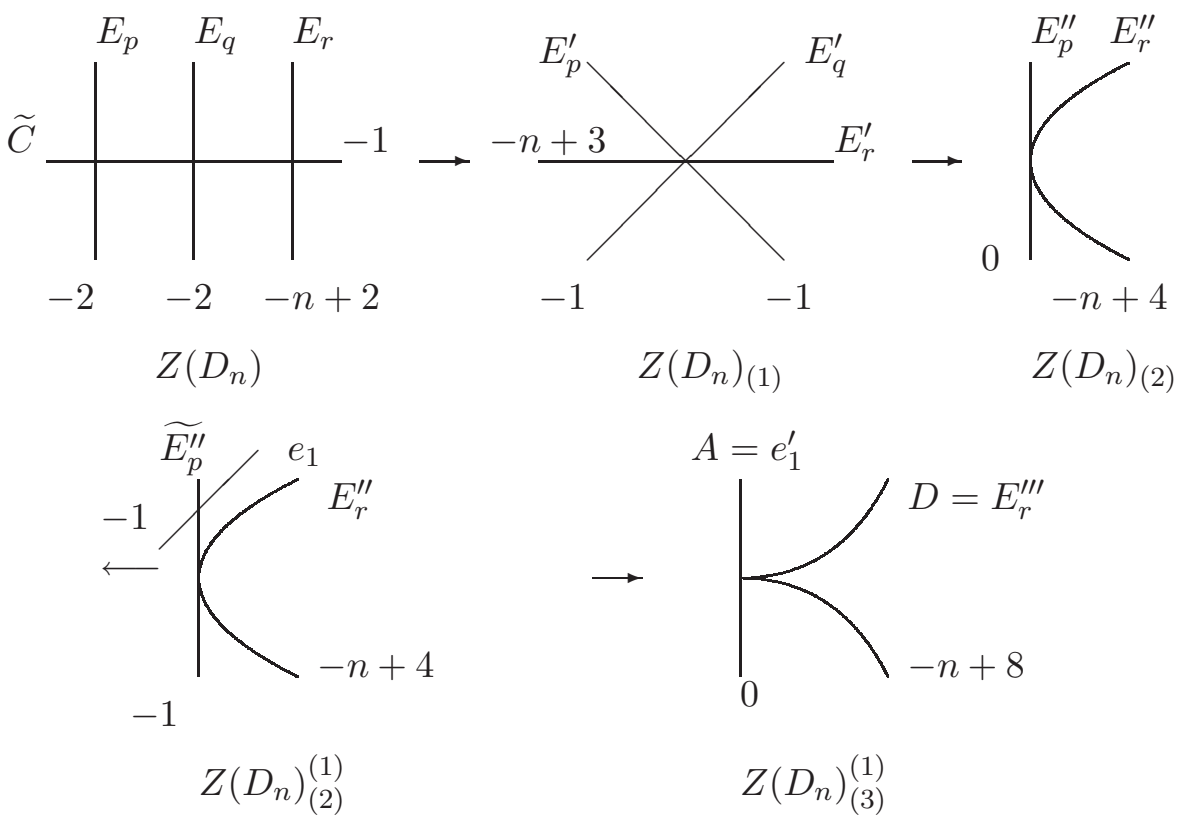

Figure 3.

Finally, we contract a maximal family of disjoint (-1)-curves away from $A$. We shall show the following:

Proposition 4.8. There is a family of disjoint (-1)-curves in $Z\left(D_{n}\right)_{(3)}^{(1)}$ such that $Z\left(D_{n}\right)_{(3)}^{(1)}$ becomes $\mathbf{C} P^{1} \times \mathbf{C} P^{1}$ after contracting those $(-1)$-curves and $A$ is identified with $\mathbf{C} P^{1} \times\{p t\}$.

Proof. We pick $J^{\prime}$ as in Lemma $4 . \overline{4}$. We may assume that $J^{\prime}$ is a small perturbation of $J$. Then, the $J$-holomorphic curve $A$ is smoothly deformed to a $J^{\prime}$-holomorphic curve $A^{\prime}$ passing through the $(2,3)$-cusp point via a small isotopy. Hereafter, we denote by $A$ the deformed curve. (In the later argument, we will consider the diffeomorphism type of the complement of a certain divisor containing $A$. Since $A$ is deformed by a small isotopy, it does not effect the argument to deal with $A^{\prime}$ instead of $A$. The same remark is valid for the curve $B$ in Theorem 4.13 below.) Then, we contract a maximal family of disjoint (-1)-curves $\epsilon_{k}$, which do not intersect $A$. Here, $\epsilon_{k}$ are $J^{\prime}$-holomorphic curves guaranteed by Lemma $4 . \overline{4}$.

Then, the resulting manifold is a ruled symplectic 4-manifold with $A$, a fiber of the ruling structure (see [23]). Since the first Betti number is 0 , the base of the fibration must be a 2 -sphere. There are two symplectic 
deformation types. They are $\mathbf{C} P^{1} \times \mathbf{C} P^{1}$ and $F_{1}$, the Hirzebruch surface of degree 1, which is diffeomorphic to $\mathbf{C} P^{2} \# \overline{\mathbf{C} P^{2}}$. Both has the second Betti number 2, hence, we find that the number of contracted $(-1)$ curves is $n$, which is positive. (Recall that $b_{2}\left(Z\left(D_{n}\right)_{(3)}^{(1)}\right)=n+2$.) If it is $\mathbf{C} P^{1} \times \mathbf{C} P^{1}$, we get the conclusion of the proposition. Otherwise, it is $F_{1}$. Pick one of $(-1)$-curves, say $\epsilon_{1}$, which is contracted to a point $p$. We make a blowing-up at the point $p$. Then, the proper transform $\tilde{f}_{p}$ of the fiber $f_{p}$ passing through the point $p$ is a $(-1)$ curve, which is disjoint from $A$ and other (-1)-curves $\left\{\epsilon_{k}\right\}_{2 \leq k \leq n}$. So, $\left\{\tilde{f}_{p}\right\} \cup\left\{\epsilon_{k}\right\}_{2 \leq k \leq n}$ is a disjoint family of symplectic $(-1)$-curves. Blowing down these symplectic $(-1)$-curves, we arrive at $\mathbf{C} P^{1} \times \mathbf{C} P^{1}$. Since $\tilde{f}_{p}$ and $\epsilon_{k}$ are disjoint from $A$, a tubular neighborhood of $A$ is not affected by the blowing-down process. Hence, $A$ has self-intersection number 0 in $\mathbf{C} P^{1} \times \mathbf{C} P^{1}$ and it is a fiber of a ruling structure.

Remark 4.9. The process of blowing up at $p$ and blowing down $\tilde{f}_{p}$ is called an elementary transformation in algebraic geometry.

Theorem 4.10. The image of the divisor $A \cup D$ in $\mathbf{C} P^{1} \times \mathbf{C} P^{1}$ consists of $\mathbf{C} P^{1} \times\{p t\}$ and a pseudo holomorphic cuspidal rational curve of bidegree $(2,2)$, i.e., representing the homology class [2,2]. Moreover, they intersect at the cusp point.

Proof. In a previous proposition, we described the blown-down manifold. Note that the singular curve $D$ represents the anti-canonical class. Thus, Lemma 4 implies that each contracted (-1)-curve intersects $D$ at a regular point transversely. Hence, the image of $D$ has the selfintersection number $-n+8+n=8$. Since the intersection number with $A=\mathbf{C} P^{1} \times\{p t\}$ is 2 , we conclude that the image of $D$ represents the homology class $[2,2]$.

q.e.d.

Case: $A_{n}$ with $(p, q, r)=(1,1,1)$ for $n=1$ and $(p, q, r)=(1,2$, $n-1)$ else.

We discuss the $A_{1}$-case separately. In this case, we contract three $(-1)$-curves $E_{p}, E_{q}, E_{r}$. Then the central curve $\widetilde{C}$ becomes a nonsingular rational curve $\bar{C}$ of self-intersection number 2. See Figure

Since there are no $(-1)$-curves in the complement of $\bar{C}$, a result of McDuff [23] asserts that the manifold is $\mathbf{C} P^{1} \times \mathbf{C} P^{1}$ and $\bar{C}$ is isotopic to the diagonal. Hence, $X$ is diffeomorphic to a disk bundle over the 2-sphere with Euler number -2 . 


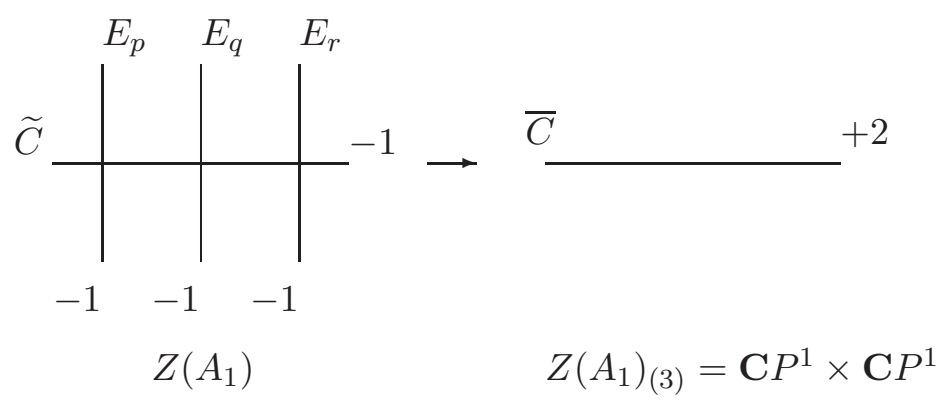

Figure 4.

Remark 4.11. The anti-canonical class is represented by $2 \bar{C}$, hence the manifold is spin. This also leads to the assertion that the manifold is $\mathbf{C} P^{1} \times \mathbf{C} P^{1}$, since it is the only symplectic deformation type of rational symplectic 4-manifolds, which are spin.

We next consider the case of $A_{n}$ with $n \geq 2$. We use the result in section 2 with $(p, q, r)=(1,2, n-1)$. Firstly, we contract the central $(-1)$-curve $\widetilde{C}$ and then, contract the image $E_{q}^{\prime}$ of $E_{q}$. The configuration consists of a rational curve $E_{p}^{\prime \prime}$ of self-intersection number 1 and a rational curve of self-intersection number $-n+3$, which are tangents to each other. Denote by $Z\left(A_{n}\right)_{(2)}$ the blown-down manifold at this stage. We blow-up $Z\left(A_{n}\right)_{(2)}$ at two points on $E_{p}^{\prime \prime}$ and, then, blow-down the proper transform $\widetilde{E}_{p}^{\prime \prime}$ of $E_{p}^{\prime \prime}$. Then, $E_{r}^{\prime \prime}$ becomes a singular rational curve $D=E_{r}^{\prime \prime \prime}$ of one $(2,3)$-cusp point and self-intersection number $-n+7$, and two exceptional curves $e_{1}$ and $e_{2}$ become two symplectically embedded spheres $A=e_{1}^{\prime}$ and $B=e_{2}^{\prime}$ with self-intersection number 0 , which intersect transversely at the cusp point of $D$. We denote by $Z\left(A_{n}\right)_{(3)}^{(2)}$ the ambient manifold at this stage. See Figure

Next, we contract a maximal family of disjoint (-1)-curves away from $A$. The following proposition is proved in a way similar to that of Proposition

Proposition 4.12. There is a family of disjoint (-1)-curves in $Z\left(A_{n}\right)_{(3)}^{(2)}$ such that $Z\left(A_{n}\right)_{(3)}^{(2)}$ becomes a ruled symplectic 4-manifold over a 2-sphere and $A$ a fiber of the ruling structure.

In this process, the contracted (-1)-curves $\epsilon_{k}$ may intersect the other rational curve $B$. However, changing the family of disjoint $(-1)$-curves, we have the following: 


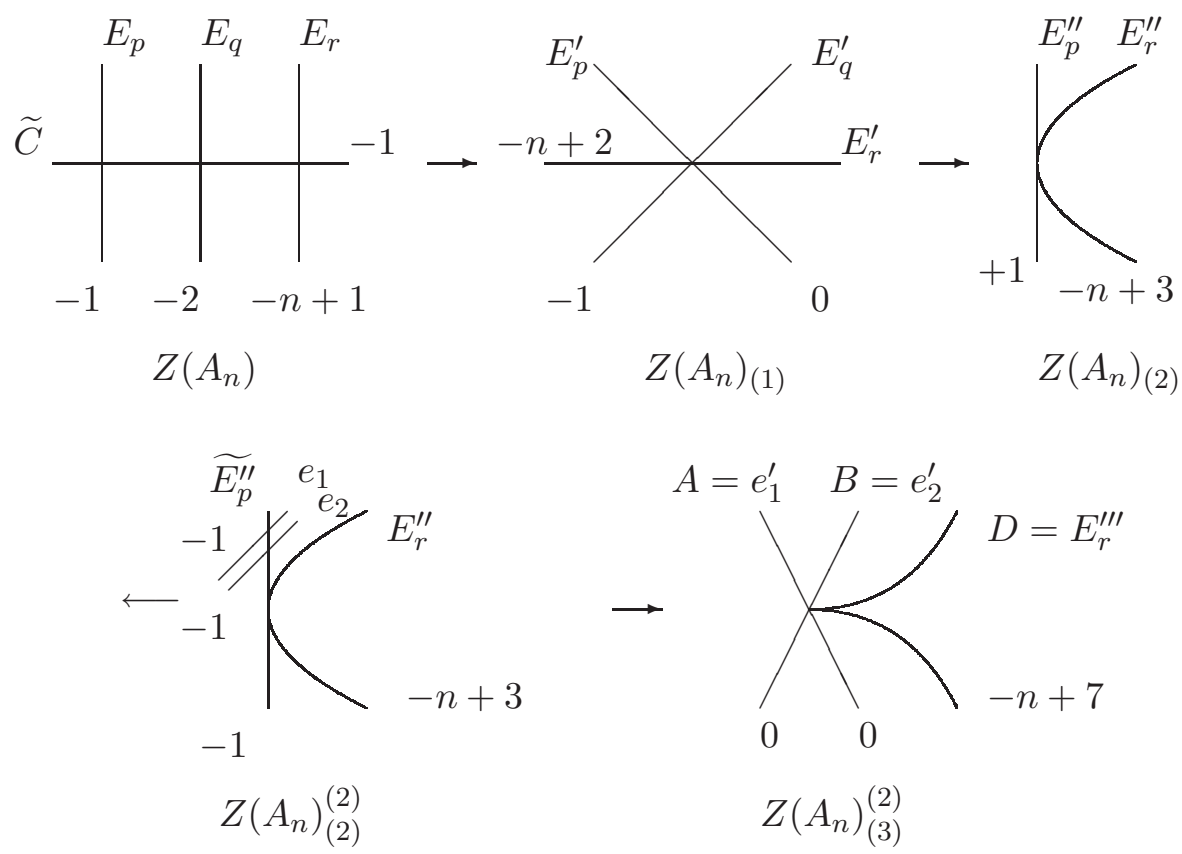

Figure 5.

Theorem 4.13. There is a family of disjoint $(-1)$-curves such that after blowing down those $(-1)$-curves $Z\left(A_{n}\right)_{(3)}^{(2)}$ becomes $\mathbf{C} P^{1} \times \mathbf{C} P^{1}$ with $A$ and $B$ being identified with $\mathbf{C} P^{1} \times\{p t\}$ and $\{p t\} \times \mathbf{C} P^{1}$ respectively. Moreover, the image of $D$ is a singular cuspidal rational curve of bidegree $(2,2)$.

Proof. From Proposition 4.12, after blowing-down a certain family of $(-1)$-curves $\epsilon_{k}, Z\left(A_{n}\right)_{(3)}^{(2)}$ becomes a ruled symplectic 4-manifold $N$ over a 2-sphere.

We note the following:

Lemma 4.14. For any $\epsilon_{k}, B$ and $\epsilon_{k}$ are disjoint or they intersect transversely at one point.

Proof. Since $A$ maps down to one of the fibers of the ruling structure, the intersection number of any fiber and the image of $B$ is +1 . Hence, the image of $B$ has no singular points, which means that $\epsilon_{k}$ intersects $B$ at most once counting with multiplicity.

q.e.d.

We return to the proof of Theorem 4.13. Suppose that some of $\epsilon_{k}$ intersect $B$. By a small smooth perturbation of the ruling structure, 
we may assume that the images of all intersection points of $B$ and $\cup \epsilon_{k}$ lie on distinct fibers on $N$. Then, we blow-up these intersection points and blow-down the proper transforms $\left\{\tilde{f}_{*}\right\}$ of the fibers $\left\{f_{*}\right\}$ passing those points. As in the previous theorem, this corresponds to changing a family of disjoint $(-1)$-curves. After blowing-down the new family of (-1)-curves which do not intersect $A$ and $B$ at all, $A$ and $B$ become symplectically embedded spheres of self-intersection number 0 in a ruled symplectic 4-manifold. This implies that the ambient manifold is $\mathbf{C} P^{1} \times \mathbf{C} P^{1}$ and $A$ and $B$ are $\mathbf{C} P^{1} \times\{p t\}$ and $\{p t\} \times \mathbf{C} P^{1}$ respectively. See Figure $\overline{\sigma_{1}^{\prime}}$ below.

The description of the image of $D$ can be obtained in a similar way as in Theorem 4.10.

q.e.d.

\section{Diffeomorphism type of $\mathbf{C} P^{2} \backslash D$}

For symplectically embedded surfaces in $\mathbf{C} P^{2}$ with small degrees, there are uniqueness results on symplectic isotopy types. (See [i-i] for degree 1 and 2 cases, 34 for the degree 3 case and [14 for more investigation. See also [i] for a related result.) Recently, Siebert and Tian proved uniqueness of symplectic isotopy type for symplectically embedded surfaces up to degree 17 [ $\overline{\mathbf{3}} \overline{\mathbf{3}}]$. We need a similar uniqueness result for pseudo holomorphic cuspidal cubic singular curves where the number of the cusp points is just 1. (See Section is. for the definition of the pseudo holomorphic cuspidal cubic curve.)

It is necessary to discuss compactness properties for pseudo holomorphic cuspidal cubic curves in order to show the uniqueness of isotopy type of such pseudo-holomorphic curves. We give a description of stable map compactification and prove transversality on a stratum containing pseudo-holomorphic cuspidal cubic curves. Barraud proved local manifold structure for moduli spaces of singular pseudo holomorphic curves under certain conditions [2]. Here, we only need to show transversality for the pseudo holomorphic curve equation for a restricted class of stable maps and give a rather straightforward proof of transversality for reader's convenience.

Let $D_{0}$ be a singular cuspidal cubic curve in $\mathbf{C} P^{2}$ with respect to the standard complex structure $J_{0}$. Let $J_{1}$ be a tame almost complex structure on $\mathbf{C} P^{2}$ and $D_{1}$ a $J_{1}$-holomorphic cuspidal cubic curve. We assume that $J_{1}$ is integrable near the cusp point $p$ on $D_{1}$. The integrability condition on $J_{1}$ will be used to guarantee pseudo-holomorphicity of proper transforms of pseudo-holomorphic curves and exceptional curves, when 

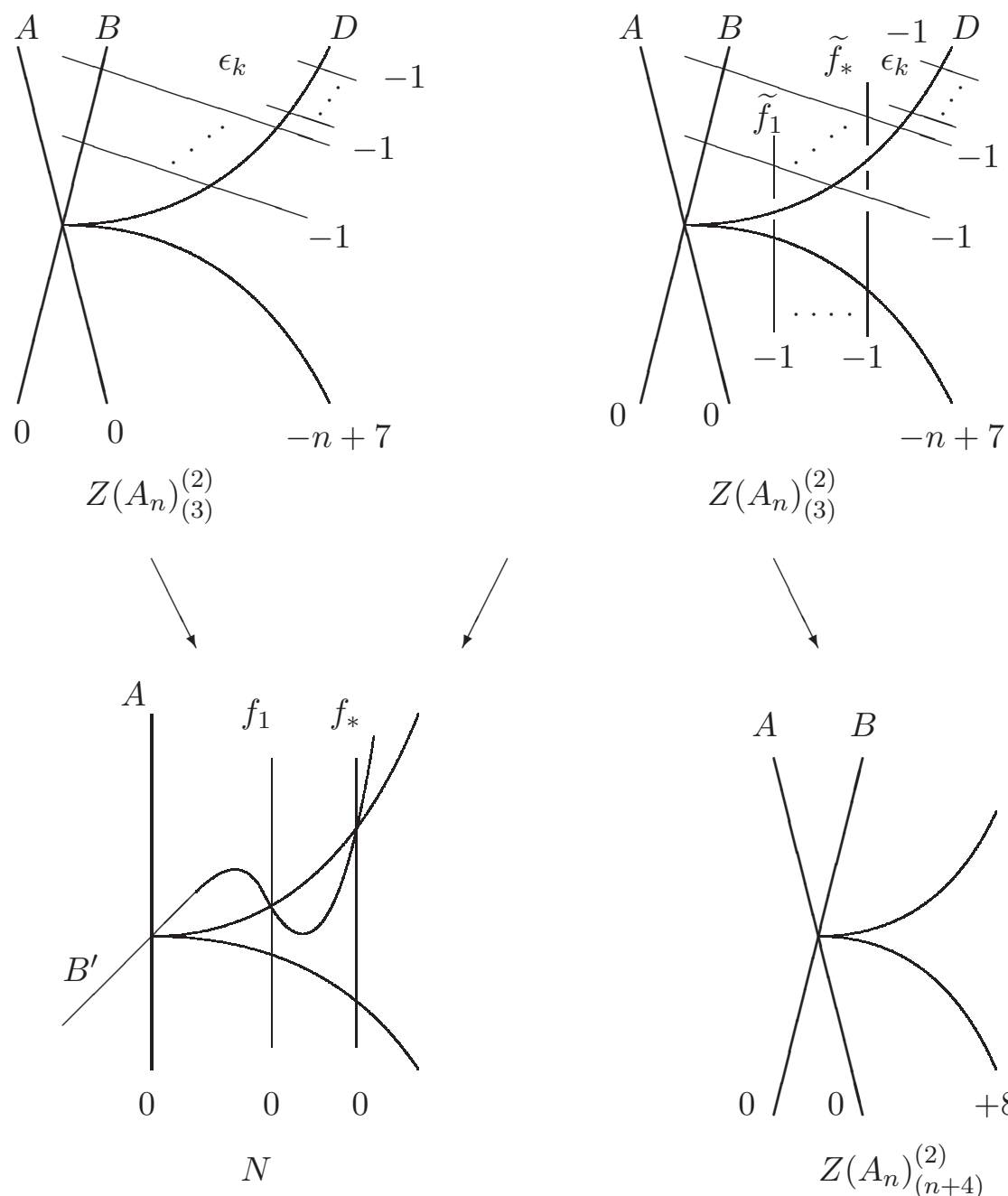

Figure 6.

we blow up $\mathbf{C} P^{2}$ at the cusp point in the proof of Theorem (Section 13$)$. By a projective transformation on $\mathbf{C} P^{2}$ if necessary, we may assume, without loss of generality, that the cusp point on $D_{0}$ coincides with $p$. The main goal of this section is to show the following:

Theorem 5.1. Let $D_{0}$ and $D_{1}$ be as above. Then, $\mathbf{C} P^{2} \backslash D_{1}$ is diffeomorphic to $\mathbf{C} P^{2} \backslash D_{0}$.

Similarly, we will prove the following:

Theorem 5.2. Let $J_{0}^{\prime}$ be the standard complex structure on $\mathbf{C} P^{1} \times$ $\mathbf{C} P^{1}$ and $D_{0}^{\prime}$ a $J_{0}^{\prime}$-holomorphic cuspidal rational curve of bidegree $(2,2)$ 
in $\mathbf{C} P^{1} \times \mathbf{C} P^{1}$. Let $D_{1}^{\prime}$ be a $J_{1}^{\prime}$-holomorphic cuspidal rational curve, which represents the same class. Here, $J_{1}^{\prime}$ is a tame almost complex structure which is integrable near the cusp point on $D_{1}^{\prime}$. Then, $\mathbf{C} P^{1} \times$ $\mathbf{C} P^{1} \backslash D_{1}^{\prime}$ is diffeomorphic to $\mathbf{C} P^{1} \times \mathbf{C} P^{1} \backslash D_{0}^{\prime}$. In particular, the diffeomorphism type is unique.

To prove Theorem 1,1, we first pick a one-parameter family of tame almost complex structures on $\mathbf{C} P^{2}$ joining the tame almost complex structure $J_{1}$ and the standard $J_{0}$ and will show that there exists a oneparameter family of $J_{t}$-holomorphic cuspidal curves with singularities of multiplicity 2 (in fact, $(2,3)$-cusp singularities, see Lemma Lemma 5.100 . By using this, we will prove Theorems Section 5.2

5.1. A family of $J_{t}$-holomorphic singular cubic curves. Let $J_{1}$ be an $\omega$-tame almost complex structure on $\mathbf{C} P^{2}$ and $D_{1}$ a $J_{1}$-holomorphic cuspidal cubic curve. Throughout this Section $5 . \overline{1}$, , we do not assume any integrability condition on $J_{1}$. So, we consider a slightly general situation in this Section. Pick a one-parameter family $\left\{J_{t}\right\}$ of $\omega$-tame almost complex structures joining the standard $J_{0}$ and $J_{1}$. For any such family $\left\{J_{t}\right\}$, we will prove in Theorem that there exists a family of $J_{t}$-holomorphic cubic curves $D_{t}$ with non-immersed points of multiplicity 2, joining $D_{0}$ and $D_{1}$. This is a main result in this Section $\overline{5}_{-1}^{1}$. To find such a one-parameter family $D_{t}$, we use the continuation method. The openness for $t \in[0,1]$ follows from transversality of the linearized operator. In dimension 4, Hofer, Lizan and Sikorav [12i] established that, when the pseudo holomorphic curve is immersed, transversality only depends on the topological type of the normal bundle. We extend the argument to a singular curve case as in [1; $\mathbf{1}]$, but with a smaller domain of the linearization operator. Since we need a family of $J_{t^{-}}$ holomorphic curves with the prescribed type of singularities, we consider the transversality problem in the space of mappings with a certain condition on the differential at a fixed point (see below). The closedness for $t \in[0,1]$ concerns compactness. For example, a sequence of cuspidal cubic curves may converge to a multiple covered curve, possibly with bubbling components. Therefore, we have to consider all possible degenerations. For this purpose, we use the stable map compactification. (For detailed discussion on stable maps, see e.g., [', $\mathbf{9}]$. In particular, see (12.1) in $[\overline{\mathbf{9}}]$ for the linearized operator of the stable map.)

Let $\mathcal{M}_{0}(3, J)$ be the moduli space of $J$-stable maps of genus 0 representing the class $3\left[\mathbf{C} P^{1}\right]$ in $\mathbf{C} P^{2}$. (Here, $J$ is an $\omega$-tame almost complex structure on $\mathbf{C} P^{2}$.) A stable map of genus 0 means that the domain semi-stable curve is of genus 0 . (Note that non-singular cubic curve is 
of genus 1. Singular cubic curves can be also seen as stable maps of genus 1. But, we do not treat them in that way.)

We stratify $\mathcal{M}_{0}(3, J)$ according to the following types.

(1) The image of the pseudo holomorphic map from the Riemann sphere is irreducible.

(1-1) It is an immersion with one double point;

(1-2) It is not an immersion and a simple pseudo holomorphic map from the Riemann sphere;

(1-3) It is not an immersion and an image of a triple covered pseudo holomorphic map from the Riemann sphere;

(2) The image of the stable map from the semi-stable curve of genus 0 consists of two irreducible components, whose degrees are 1 and 2 respectively.

(2-1) Both components are simple, that is, embedded;

(2-2) The degree 2 component is a double cover of a degree 1 curve;

(3) The image of the stable map from the semi-stable curve of genus 0 consists of at most three irreducible components, whose degrees are 1.

(3-1) The domain of the stable map consists of three copies of the Riemann sphere;

(3-2) The domain of the stable map consists of four copies of the Riemann sphere.

In type (2-1), there are two possibilities.

(2-1-1) The degree 2 component and the degree 1 component intersects transversely at two points (One of them corresponds to the node in the domain of the stable map.);

(2-1-2) The degree 2 component and the degree 1 component are tangent to each other at a point.

In type (3), the domain of the stable map is a semi-stable curve of genus 0, i.e., a tree of copies of the Riemann sphere. The stability condition implies that the holomorphic map is not constant on the components corresponding to the exterior vertices of the tree. Hence, there are at most three exterior vertices. In case (3), there are two possibilities. Namely, (3-1) and (3-2). We remark that the stable maps of type (3-2) are ones from the semi-stable curve consisting of four irreducible components of genus 0 (see Figure i i i ), three curves of degree 1 meet, in the image, at a common point, to which the central fourth component is mapped constantly. Further, we remark that in type (1-1) the number of the double points is necessarily one, because the adjunction formula implies that the virtual genus of the image is 
1 , which is bigger than the genus of the domain of the stable map by just 1 .

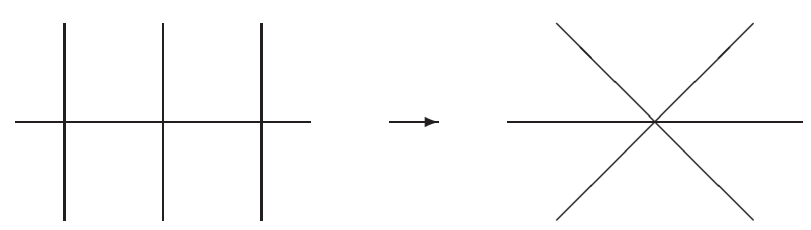

Figure 7.

Although, we can introduce a finer stratification on $\mathcal{M}_{0}(3, J)$, the stratification above is enough for our purpose. We note that the pseudo holomorphic cuspidal cubic curve in $\mathbf{C} P^{2}$, which we are interested in, belongs to the stratum of type (1-2) by the following lemma.

Lemma 5.3. The image of the pseudo holomorphic map of type (1-2) has only one singular point with multiplicity 2.

Proof. We denote the image by $D$. Since it is not an immersion, it has a singular point. Since the virtual genus of $D$ is 1 and the genus of the domain of the pseudo holomorphic map is 0 , we find that $D$ has only one singular point $p$. To prove the lemma, it is enough to show that

$$
\min _{l}\left\{l \cdot{ }_{p} D \mid l \text { is a pseudo holomorphic line in } \mathbf{C} P^{2} \text { through } p\right\}=2 \text {, }
$$

where $l \cdot{ }_{p} D$ is the intersection number of $l$ and $D$ at $p$. Since the intersection number of two pseudo holomorphic curves in an almost complex 4-manifold is always positive [24], we find that the number in the left hand side above is at most 3 . On the other hand, $l \cdot{ }_{p} D=1$ implies that $l$ and $D$ intersect transversely at $p$ by [2-i $\mathbf{4}]$. This contradicts to that $p$ is the singular point. If it is 3 , then $l \cdot p D=3$ for all pseudo holomorphic lines $l$ through $p$. We pick a point $q$ on $D$ which is different from the singular point $p$. By Gromov's theorem [11], there exists a pseudo holomorphic line $l$ through $p$ and $q$. In this case, we have $l \cdot D \geq 4$, which is a contradiction. Thus, Lemma 5.3 is proved.

Although, we have to deal with the stratum of type (1-2) only, there is no guarantee that the closure of this stratum is a nice topological space such as an orbifold. Therefore, we firstly list up the strata appearing the closure of the stratum of type (1-2) in the moduli space $\mathcal{M}_{0}(3, J)$. 
Obviously, the closure of the stratum of type (1-2) does not intersect the stratum of type (1-1). If the image of the stable map in the stratum (3-1) consists of distinct three lines, the three components in the domain are mapped to three pseudo holomorphic lines with three double points. Hence, after smoothing two of three double points, the glued pseudo holomorphic curve must have one double point, which never occurs in type (1-2). Thus, the closure of the stratum of type (1-2) intersects the stratum of type (3-1) along the locus, where the image consists of at most two pseudo holomorphic lines. Similarly, the closure of the stratum of type (1-2) does not intersect the strata of types (2-1-1). Hence, the closure of the stratum of type (1-2) is contained in the union of the strata of types (1-2), (1-3), (2-1-2), (2-2), (3-1) and (3-2) (see examples below). We will impose some further constraints on the pseudo holomorphic curves in $\mathcal{M}_{0}(3, J)$ to exclude the possibilities that a sequence of pseudo holomorphic curves in the stratum of type (1-2) converges to any element in the union of the strata of types (1-3), $(2-1-2),(2-2),(3-1),(3-2)$. It is clear that the closure of the stratum of type (1-2) intersects the strata of type (1-3) and type (2-2). For the strata of type (2-1-2) and type (3-2), see the following:

\section{Example 5.4.}

(1) Consider a one-parameter family of cuspidal cubic curves $y^{2} z-$ $x^{2} y-\epsilon x^{3}=0$ with $\epsilon \neq 0$. Each of them has a $(2,3)$-cusp singularity at $[0,0,1]$. As $\epsilon$ tends to 0 , the limit curve splits into the union of $y=0$ and $y z-x^{2}=0$. Thus, the closure of the stratum of type (1-2) intersects the stratum of type (2-1-2).

(2) Consider a one-parameter family of cuspidal cubic curves $x^{3}-$ $x y^{2}-\epsilon y^{2} z=0$ with $\epsilon \neq 0$. Each of them has a $(2,3)$-cusp singularity at $[0,0,1]$. As $\epsilon$ tends to zero, the limit consists of three lines $x=0, x+y=0$ and $x-y=0$. As a stable map, there appears another rational component, which is mapped to $[0,0,1]$. Thus, the closure of the stratum of type (1-2) intersects the stratum of type (3-2).

Pick distinct 6 points $p=q_{1}, \ldots, q_{6}$ on $\mathbf{C} P^{2}$. We consider the moduli space

$$
\mathcal{M}_{0}\left(3, J ; p ; q_{2}, \ldots, q_{6}\right)
$$

of $J$-holomorphic rational cubic curves with a non-immersed point at $p=q_{1}$ and passing through $q_{2}, \ldots, q_{6}$. To do so, we consider the moduli space $\mathcal{M}_{0,6}(3, J)$ of stable maps of genus zero, with 6 marked points $z_{1}, \ldots, z_{6}$ and of degree 3 . Throughout this Section 1 , we arrange $z_{1}, z_{2}$ 
and $z_{3}$ at $[1,0],[0,1],[1,1]$, respectively, by $\operatorname{Aut}\left(\mathbf{C} P^{1}\right)$. Let $e$ be a nonzero tangent vector at $z_{1}$. We consider the following evaluation map:

$$
\text { ev }: \mathcal{M}_{0,6}(3, J) \rightarrow T \mathbf{C} P^{2} \times \mathbf{C} P^{2} \times \cdots \times \mathbf{C} P^{2}
$$

given by $\left(\phi,\left(z_{1}, z_{2}, \ldots, z_{6}\right)\right) \mapsto\left(d \phi(e), \phi\left(z_{2}\right), \ldots, \phi\left(z_{6}\right)\right)$. Then we have

$$
\mathcal{M}_{0}\left(3, J ; p ; q_{2}, \ldots, q_{6}\right)=\mathbf{e v}^{-1}\left((p, 0), q_{2}, \ldots, q_{6}\right) .
$$

For any $\left(\phi,\left(z_{1}, \ldots, z_{6}\right)\right) \in \mathcal{M}_{0}\left(3, J ; p ; q_{2}, \ldots, q_{6}\right)$ with $\phi: \mathbf{C} P^{1} \rightarrow \mathbf{C} P^{2}$ of type 1-2), we have the following:

Proposition 5.5. The moduli space $\mathcal{M}_{0}\left(3, J ; p ; q_{2}, \ldots, q_{6}\right)$ is an oriented smooth 0-dimensional manifold around $\left(\phi,\left(z_{1}, \ldots, z_{6}\right)\right)$.

Proof. We consider the space of singular cubic pseudo holomorphic curves with a non-immersed point at $p=q_{1}$ and passing through $q_{2}, \ldots$, $q_{6}$. We adapt the framework in [i-i, to deal with non-immersed pseudo holomorphic curves. Pick $p>\overline{2}$ and consider the $W^{2, p}$ completion $\mathcal{X}$ of the space of quadruples $\left(\phi, z_{4}, z_{5}, z_{6}\right)$, where $\phi: \mathbf{C} P^{1} \rightarrow \mathbf{C} P^{2}$ is a smooth map representing the homology class $3\left[\mathbf{C} P^{1}\right]$ such that $\phi\left(z_{i}\right)=q_{i}, i=1, \ldots, 6, d \phi(e)=0$. Note that $d \phi$ vanishes at $z_{1}$, if $\phi$ in this space is a pseudo holomorphic curve. The tangent space of $\mathcal{X}$ at $\left(\phi,\left(z_{4}, z_{5}, z_{6}\right)\right)$ is the space of quadruples $\left(\xi, v_{4}, v_{5}, v_{6}\right)$, where $\xi \in \Gamma\left(\phi^{*} T \mathbf{C} P^{2}\right)$ and $v_{i} \in T_{z_{i}} \mathbf{C} P^{1}$, such that $\xi\left(z_{i}\right)=0$ for $i=1,2,3$, $\xi\left(z_{i}\right)+d \phi\left(v_{i}\right)=0$ for $i=4,5,6$ and $\nabla_{e} \xi=0$.

Let $\left(\phi,\left(z_{4}, z_{5}, z_{6}\right)\right)$ be a $J$-holomorphic cubic curve with a non-immersed point at $p=q_{1}$ and passing through $q_{i}$. By the adjunction formula, each rational cubic pseudo holomorphic curve has either a unique double point (node) or a unique non-immersed point of multiplicity 2, which implies that $\phi$ is an immersion near $z_{2}, \ldots, z_{6}$. Thus, the linearization of the pseudo holomorphic curve equation in this setting is

$$
D \bar{\partial}: W \rightarrow W^{1, p}\left(\Omega^{0,1}\left(\mathbf{C} P^{1}, \phi^{*} T \mathbf{C} P^{2}\right)\right),
$$

where $W$ is the $W^{2, p}$-completion of the space of $\xi \in \Gamma\left(\phi^{*} T \mathbf{C} P^{2}\right)$ such that $\nabla_{e} \xi=0, \xi\left(z_{i}\right)=0, i=1,2,3$ and $\xi\left(z_{i}\right) \in d \phi\left(T_{z_{i}} \mathbf{C} P^{1}\right), i=4,5,6$. $D \bar{\partial}$ is a first order partial differential operator with the same principal symbol as the Dolbeault operator with coefficients in $\phi^{*} T \mathbf{C} P^{2}$.

Since $\phi$ is not an immersion, the image of $T \mathbf{C} P^{1}$ by $d \phi$ is not a subbundle of $\phi^{*} T \mathbf{C} P^{2}$. However, we can extend the image of $d \phi$ to a subline bundle of $\phi^{*} T \mathbf{C} P^{2}$ as follows. Firstly, pick a complex linear connection $\nabla$ on $T \mathbf{C} P^{2}$ such that $N=8 T$, where $N$ is the Nijenhuis tensor of $J$ and $T$ is the torsion tensor of $\nabla$. (See [1 6] Theorem 3.4 in Chapter IX). Since the base space is a Riemann surface, the pull-back connection induces a structure of a holomorphic vector bundle. Now, we show 
the following lemma. (A similar result is well-known for harmonic maps between Kähler manifolds.)

Lemma 5.6. The $(1,0)$-part $\partial \phi$ of the differential $d \phi$ is a holomorphic section of $\Lambda^{1,0}\left(\mathbf{C} P^{1} ; \phi^{*} T \mathbf{C} P^{2}\right)$, if $\phi$ is J-holomorphic.

Proof. We take the standard connection, i.e., a torsion free unitary connection on $\mathbf{C} P^{1}$. Denote by $j$ the complex structure on $\mathbf{C} P^{1}$. For each point $z \in \mathbf{C} P^{1}$, we choose a local unit vector field e such that $\nabla \mathbf{e}(z)=0$. Then, $(\mathbf{e}, j \mathbf{e})$ is a unitary frame field on $\mathbf{C} P^{1}$, whose covariant derivatives vanishes at $z$. Write $\epsilon=\mathbf{e}^{*}+\sqrt{-1}\left(j \mathbf{e}^{*}\right)$. Then, we have

$$
\partial \phi=(d \phi(\mathbf{e})-\sqrt{-1} d \phi(j \mathbf{e})) \otimes \epsilon .
$$

Compute the $(0,1)$-part of the covariant derivative of $\partial \phi$.

$$
\nabla^{0,1} \partial \phi=\left(\nabla_{\mathbf{e}}+\sqrt{-1} \nabla_{j \mathbf{e}}\right) \partial \phi \otimes \bar{\epsilon}
$$

Note that $T(d \phi(\mathbf{e}), d \phi(j \mathbf{e}))=T(d \phi(\mathbf{e}), J d \phi(\mathbf{e}))=N(d \phi(\mathbf{e}), J d \phi(\mathbf{e})) / 8$, the last quantity of which is identically zero. Hence, we have $\nabla_{\mathbf{e}} d \phi(j \mathbf{e})=$ $\nabla_{j \mathbf{e}} d \phi(\mathbf{e})$ at $z$, since $[\mathbf{e}, j \mathbf{e}]$ vanishes at $z$. Using this equality and identifying $T^{*} \mathbf{C} P^{1^{(1,0)}} \otimes T^{*} \mathbf{C} P^{1^{(0,1)}}$ with $T^{*} \mathbf{C} P^{1^{(0,1)}} \otimes T^{*} \mathbf{C} P^{1^{(1,0)}}$, we have

$$
\nabla^{0,1} \partial \phi=\nabla^{1,0} \bar{\partial} \phi
$$

The right hand side vanishes, since $\phi$ is $J$-holomorphic.

q.e.d.

We consider the invertible sheaf defined by the image of

$$
\partial \phi: \mathcal{O}\left(T \mathbf{C} P^{1}\right) \rightarrow \mathcal{O}\left(\phi^{*} T \mathbf{C} P^{2}\right) .
$$

We extend it to a holomorphic subline bundle. Note that the nonconstant simple pseudo holomorphic map $\phi: \mathbf{C} P^{1} \rightarrow \mathbf{C} P^{2}$ can be locally expanded around the singular point in the following form:

$$
\phi(z)=\left(z^{k}, z^{m}\right)+O(m+1),
$$

where $m>k, k$ does not divide $m$ and $O(m+1)$ denotes a function of $z$ and $\bar{z}$ which vanishes to order $m$. (See Proposition 2.6 in [24].) Therefore, Lemma is 2 , thus the order of $d \phi$ at the singular point is 1 . Then, the argument in page 516 in [i10 $\mathbf{0}]$ implies that $\phi^{*} T \mathbf{C} P^{2}$ contains the line bundle $L_{1}=$ $T \mathbf{C} P^{1} \otimes \mathcal{O}\left(z_{1}\right)$ of degree 3 with a quotient line bundle $L_{2}$ of degree 6 :

$$
0 \rightarrow L_{1} \rightarrow \phi^{*} T \mathbf{C} P^{2} \rightarrow L_{2} \rightarrow 0 .
$$

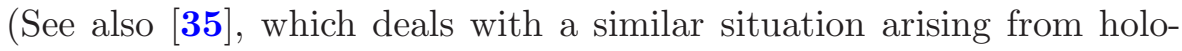
morphic maps.)

Note that, for $\xi \in W^{2, p}\left(L_{1}\right), D \bar{\partial} \xi \in W^{1, p}\left(\Omega^{0,1}\left(\mathbf{C} P^{1}, L_{1}\right)\right)$. Therefore, it is enough to show surjectivity of the following two maps: 
(a) $D \bar{\partial}: W \cap W^{2, p}\left(L_{1}\right) \rightarrow W^{1, p}\left(\Omega^{0,1}\left(\mathbf{C} P^{1}, L_{1}\right)\right)$.

(b) The composition $W \cap W^{2, p}\left(L_{2}\right) \rightarrow W \rightarrow W^{1, p}\left(\Omega^{0,1}\left(\mathbf{C} P^{1}, \phi^{*} T \mathbf{C} P^{2}\right)\right)$ $\rightarrow W^{1, p}\left(\Omega^{0,1}\left(\mathbf{C} P^{1}, L_{2}\right)\right)$, where the first map is the inclusion, the second is $D \bar{\partial}$ and the last is the one induced by the projection $\phi^{*} T \mathbf{C} P^{2} \rightarrow L_{2}$.

In order to prove surjectivity for these operators, it is enough to prove the following:

Lemma 5.7. Let $L$ be a holomorphic line bundle over $\mathbf{C} P^{1}$ with $c_{1}(L) \geq k$ and $\mathcal{D}$ a first order differential operator with the same symbol as the Dolbeault operator. Let $z_{1}, \ldots, z_{k}$ be distinct points on $\mathbf{C} P^{1}$ and $e$ a non-zero tangent vector at $z_{1}$. Then, $\mathcal{D}$ is surjective from $\left\{\xi \in \Gamma(L) \mid \xi\left(z_{i}\right)=0, i=1, \ldots, k\right.$, and $\left.\nabla_{e} \xi=0\right\}$ to $\Omega^{0,1}(L)$.

We remark that in the case (a), we have the restriction on the domain such that $\nabla_{e} \xi=0, \xi\left(z_{i}\right)=0, i=1,2,3$, and in the case (b), we have $\nabla_{e} \xi=0, \xi\left(z_{i}\right)=0, i=1, \ldots, 6$. Thus, the surjectivity of these maps follows from above lemma for $k=3$ and 6 , respectively.

Proof of Lemma $\overline{5}$. Let $\xi$ be a solution of $\mathcal{D} \xi=0$ such that $\xi\left(z_{1}\right)=0$ and $\nabla_{e} \xi=0$. Note that these conditions imply that the 1-jet of $\xi$ vanishes at $z_{1}$.

Pick a complex linear connection on $L$ and write the operator $\mathcal{D}$ as

$$
\mathcal{D}=\bar{\partial}+B_{l}+B_{a},
$$

where $B_{l}$ and $B_{a}$ are the complex linear part and the anti-complex linear part of the 0 -th order operator $\mathcal{D}-\bar{\partial}$, respectively.

We shall show that the local intersection index of $\xi$ with the zero section at $p$ is at least 2. Denote by $w$ a local holomorphic coordinate of $\mathbf{C} P^{1}$ with $w\left(z_{1}\right)=0$. Since the difference of $\mathcal{D}$ and the Dolbeault operator is a 0 -th order operator, we have $\bar{\partial} \xi$ vanishes at $z_{1}$. Hence, $\xi / w$ is locally a $W^{1, p}$-function. Write $\eta=\xi / w$, which satisfies the following differential equation.

$$
\bar{\partial} \eta+B_{l}(\eta)+\frac{\bar{w}}{w} B_{a}(\eta)=0
$$

Note that $\bar{w} / w$ is locally $L^{\infty}$. Therefore, we can apply the Carleman similarity principle (Appendix A.6 in [i] $\left.\mathbf{i}_{1}\right]$ ) to this situation and conclude that the intersection index of $\eta$ and the zero section at $z_{1}$ is positive, if $\eta\left(z_{1}\right)=0$. Recall that the 1 -jet of $\xi$ vanishes at $z_{1}$, hence $\eta$ vanishes at $z_{1}$. This implies that the intersection index of $\xi$, which equals $w \eta$ near $z_{1}$, and the zero section at $z_{1}$ must be at least 2 .

Since the index of the real Fredholm operator $\mathcal{D}$ from

$$
\left\{\xi \in \Gamma(L) \mid \xi\left(z_{i}\right)=0, i=1, \ldots, k, \text { and } \nabla_{e} \xi=0\right\}
$$


to $\Omega^{0,1}(L)$ is $2 c_{1}(L)-2 k$, it is sufficient to show that the dimension of the kernel does not exceed $2 c_{1}(L)-2 k$.

Let us assume the contrary. Pick $d=c_{1}(L)-k$ distinct points $w_{1}, \ldots, w_{d}$ on $\mathbf{C} P^{1} \backslash\left\{z_{i}\right\}$. By assumption, there are at least $2 d+1$ linearly independent sections $s_{1}, \ldots, s_{2 d+1}$ satisfying $\mathcal{D} s_{i}=0, s_{i}\left(z_{j}\right)=0$, $j=1, \ldots, k$ and $\nabla_{e} s_{i}=0$. Then, we can find real numbers $\lambda_{1}, \ldots, \lambda_{2 d+1}$, which contains non-zero numbers, such that the linear combination $s=\sum \lambda_{i} s_{i}$ vanishes at $w_{1}, \ldots, w_{d}$ as well as $z_{i}$. Note that $s$ is a non-zero section and all zeros contribute positively to the intersection number of $s$ and the zero section [1]2in. Since we have shown that the intersection index at $z_{1}$ is at least 2 , the self-intersection number, which is $c_{1}(L)$ must be at least $d+k+1=c_{1}(L)+1$. This is a contradiction. q.e.d.

We have proved that the moduli space $\mathcal{M}_{0}\left(3, J ; p ; q_{2}, \ldots, q_{6}\right)$ is a smooth manifold. Since the family of linearization operators are deformed to a family of complex linear Fredholm operators, the moduli space carries a natural orientation. The index computation shows that its dimension is 0 . We have finished the proof of Proposition

Now, we are going to state and prove the main theorem in this Section 5.1 .1 Recall that $D_{1}$ is a $J_{1}$-holomorphic cuspidal cubic curve and $D_{0}$ is a cuspidal cubic curve with respect to the standard complex structure $J_{0}$ on $\mathbf{C} P^{2}$. We do not assume any integrability condition on $J_{1}$ here. We denote by $p$ the cusp point on $D_{1}$. We may assume that the cusp point on $D_{0}$ coincides with $p$. By Lemma 5.2, they belong to the stratum of type (1-2).

Theorem 5.8. Let $D_{1}$ and $D_{0}$ be pseudo holomorphic curves as above. Pick a one-parameter family $\left\{J_{t}\right\}$ of $\omega$-tame almost complex structures joining $J_{0}$ and $J_{1}$. Then, there is a one-parameter family of singular cubic pseudo-holomorphic curves $D_{t}$ joining $D_{0}$ and $D_{1}$ such that for each $t, D_{t}$ has only one non-immersed point of multiplicity 2 and it is fixed at $p$ in $\mathbf{C} P^{2}$.

Proof. We show the following:

Lemma 5.9. Let $\left\{J_{t}\right\}$ be as in Theorem 5.8 . Then, we can choose $p=q_{1}, q_{2}, \ldots, q_{6}$ on $\mathbf{C} P^{2}$ so that the following three conditions are satisfied for each $t \in[0,1]$.

(1) There is no collection of three $J_{t}$-holomorphic curves of degree 1 with a triple intersection point, whose union contains $q_{1}, \ldots, q_{6}$, especially, these six points do not lie on a line or the union of two lines. 


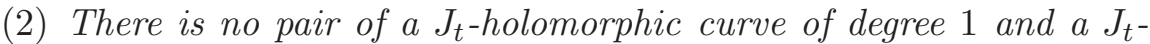
holomorphic curve of degree 2 , which are tangents at $p=q_{1}$ and contain $q_{2}, \ldots, q_{6}$.

(3) There is a $J_{0}$-holomorphic cuspidal cubic curve with a cusp at $p$ and passing through $q_{2}, \ldots, q_{6}$.

We complete the proof of Theorem 5.8 assuming Lemma 5.9 .9 . Firstly, we note that $\bigcup_{0 \leq t<1}\{t\} \times \mathcal{M}_{0}\left(3, J_{t} ; p ; q_{2}, \ldots, q_{6}\right)$ is compact. Assume the contrary and $\left\{\left(t_{j}, \phi_{j}, z_{4, j}, z_{5, j}, z_{6, j}\right)\right\}$ is not convergent in

$$
\bigcup_{0 \leq t \leq 1}\{t\} \times \mathcal{M}_{0}\left(3, J_{t} ; p ; q_{2}, \ldots, q_{6}\right) .
$$

We may assume that $t_{j}$ converges to $t_{0}$. After taking a suitable subsequence, $\phi_{j}$ converges, as stable maps, to an element $\phi_{0}$ in one of the stratum strata (1-3), (2-1-2), (2-2), (3-1), (3-2) passing through $q_{i}$. Moreover, we can see the following:

(1) If $\phi_{0}$ is in the stratum of type (2-1-2), $p$ is the point, where two components of $\phi_{0}$ are tangent;

(2) If $\phi_{0}$ is in the stratum of type (3-1), the image of $\phi_{0}$ consists of at most two $J_{t_{0}}$-holomorphic lines.

(3) If $\phi_{0}$ is in the stratum of type (3-2), $p$ is the common intersection point of three components of $\phi_{0}$.

By Lemma $5 . \overline{5}, 9$, we can exclude these possibilities. If $\phi_{0}$ is in one of the stratum of type (1-3), (2-2), the six points $q_{i}$ must lie on the union of at most two $J_{t}$-holomorphic curves of degree 1 , hence these possibilities are also excluded by Lemma $\overline{5} .9$. Therefore, $\bigcup_{0 \leq t<1}\{t\} \times$ $\mathcal{M}_{0}\left(3, J_{t} ; p ; q_{2}, \ldots, q_{6}\right)$ is compact. Proposition 5.5 and its proof imply that it is a 1-dimensional manifold and the first factor projection to $[0,1]$ is submersive, i.e., the rank of its differential is 1 everywhere, if it is not empty. In other words, $\bigcup_{0 \leq t \leq 1}\{t\} \times \mathcal{M}_{0}\left(3, J_{t} ; p ; q_{2}, \ldots, q_{6}\right)$ is a product cobordism.

Finally, we claim that it is not empty. The third condition in Lemma $J_{0}$ is an integrable complex structure, transversality (Proposition implies that all elements in $\mathcal{M}_{0}\left(3, J_{0} ; p ; q_{2}, \ldots, q_{6}\right)$ have positive sign with respect to the natural orientation. Therefore, it is not null orientedcobordant and $\bigcup_{0 \leq t \leq 1}\{t\} \times \mathcal{M}_{0}\left(3, J_{t} ; p ; q_{2}, \ldots, q_{6}\right)$ is non-trivial.

Moreover, any two cuspidal cubic curves with respect to the standard complex structure $J_{0}$ are projectively equivalent (see, for example, [2.25] p.128). Thus, we can further deform the cuspidal cubic curve to a standard one, e.g., $\left\{x^{3}+y^{2} z=0\right\}$ in $\mathbf{C} P^{2}$. Thus, this gives a smooth one 
parameter family joining $D_{1}$ and $D_{0}$ through pseudo-holomorphic cubic curves with non-immersed points of multiplicity $2 . \quad$ q.e.d.

Proof of Lemma $\overline{5}$. . Denote by $\bigcup_{1 \leq t \leq 1}\{t\} \times \mathcal{M}_{0,6}\left(3_{3-2}, J_{t}\right)$ the moduli space of $J_{t}$-stable maps in the stratum (3-2) with 6 marked points. The real dimension of this space is 23 . Thus, the set of $\left\{q_{1}, \ldots, q_{6}\right\}$ lying on the union of three $J_{t}$-holomorphic curves of degree 1 for some $t$ is a 23-dimensional subspace of the product of 6 copies of $\mathbf{C} P^{2}$. The evaluation map at $z_{1}$ is easily seen to be a submersion. Hence we get a 19-dimensional subspace of the product of 5 copies of $\mathbf{C} P^{2}$, when the condition that $q_{1}=p$ is required.

We next consider the stratum (2-1-2). To do this, we denote by $\mathcal{M}_{0,1^{*}}(1, J)$ (resp. $\left.\mathcal{M}_{0,1^{*}}(2, J)\right)$ the moduli space of genus $0 J$-holomorphic curves of degree 1 (resp. degree 2) with a marked point $z \in \mathbf{C} P^{1}$ and a non-zero tangent vector $e \in T_{z} \mathbf{C} P^{1}$. Let $\mathbf{e v}_{i}(i=1,2)$ be the evaluation map $\mathbf{e v}_{i}(\phi, z, e)=d \phi(e)$ for each of them. The transversality for $\mathcal{M}_{0,1^{*}}(1, J)$ and $\mathcal{M}_{0,1^{*}}(2, J)$ is well-known by [1]1]. Here, $\mathcal{M}_{1^{*}}(2, J)$ is the moduli space before taking the stable map compactification. Similarly, we can show that the evaluation maps $\mathbf{e v}_{i}$ are of maximal rank. We take the fiber product of $\mathcal{M}_{0,1^{*}}(1, J)$ and $\mathcal{M}_{0,1^{*}}(2, J)$ over $T \mathbf{C} P^{2}$. The fiber product is real 14-dimensional. Note that $\mathbf{C}^{*}$ acts on each of $\mathcal{M}_{0,1^{*}}(i, J)(i=1,2)$ by complex multiplication to $v \in T_{z} \mathbf{C} P^{1}$. Note also that pseudo holomorphic curves in $\mathcal{M}_{0,1^{*}}(i, J)(i=1,2)$ are embedded, $\mathbf{C}^{*}$ acts freely on the fiber product. Thus, it has a $\mathbf{C}^{*}$-bundle structure so that its quotient by the $\mathbf{C}^{*}$ action is the moduli space of configuration of two $J_{t}$-holomorphic curves of degree 1 and 2 , which are tangents to each other. After adding 5 marked points $z_{2}, \ldots, z_{6}$, the moduli space becomes real 22 dimensional. (Note that the "first" marked point is automatically determined as the tangent point.) Now, we vary $t \in[0,1]$. Then, we have the moduli space of real 23-dimensions. Thus, the set of $\left\{q_{1}, \ldots, q_{6}\right\}$ lying on the union of two $J_{t}$-holomorphic curves of degree 1 and 2, which are tangents at $q_{1}$, for some $t$ is a 23dimensional subspace of the product of 6 copies of $\mathbf{C} P^{2}$. Again, by requiring the condition that $q_{1}=p$, we get a 19 dimensional subspace of the product of 5 copies of $\mathbf{C} P^{2}$.

Pick a cuspidal cubic curve with a cusp at $p$ with respect to the standard complex structure $J_{0}$ and 5 distinct points $q_{2}, \ldots, q_{6}$ on it. Denote by $\mathcal{M}_{0,1+5}\left(3_{1-2}, J_{0}\right)$ the moduli space of cuspidal cubic curves with 6 marked point $\left\{z_{1}, \ldots, z_{6}\right\}$ such that $z_{1}$ is the unique singular point. Note that transversality established in the proof of Proposition $\overline{5} . \overline{1} \mathrm{im}-$ plies that the evaluation map

$$
\text { ev }: \mathcal{M}_{0,1+5}\left(3_{1-2}, J_{0}\right) \rightarrow T \mathbf{C} P^{2} \times \mathbf{C} P^{2} \times \cdots \times \mathbf{C} P^{2}
$$


is of maximal rank (24 over reals). When we require the condition that $q_{1}=p$, we get a 20 dimensional family of $\left\{q_{2}, \ldots, q_{6}\right\}$ in the product of 5 copies of $\mathbf{C} P^{2}$. Comparing dimensions of the image of evaluation maps, we can choose $\left\{q_{1}, \ldots, q_{6}\right\}$, which satisfies three conditions in Lemma 5.9.

q.e.d.

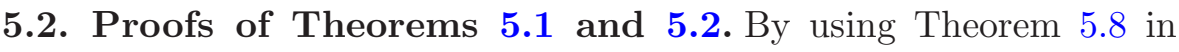
Section $\overline{5} .1$, we will prove Theorems $5.1,1$ and Theorems 5.12 and

Firstly, we recall that in Section 4 ' 2 we take the tame almost complex structure on the compactification $\bar{Z}(\bar{\Gamma})$ such that it is integrable near the compactifying divisor. Corresponding to this, we take a tame almost complex structure $J_{1}$ on $\mathbf{C} P^{2}$ so that $D_{1}$ is a $J_{1}$-holomorphic cuspidal cubic curve and $J_{1}$ is integrable near the cusp point $p$, throughout this Section 5.2 In this situation, we note that the singular point in the image of the pseudo holomorphic map of type (1-2) has not only multiplicity 2 (Lemma 5.3$)$, but is also a $(2,3)$-cusp singularity as follows.

Lemma 5.10. Around the singular point $P$, the pseudo holomorphic map of type (1-2) can be written as $z \mapsto\left(z^{2}, z^{3}\right)+O(4)$.

Proof. By Lemma 5.3 and Proposition 2.6 in [24], we can write the map germ around the cusp point as $z \mapsto(u, v)=\left(z^{2}, z^{k}\right)+O(k+1)$ for some $k \geq 3$. Suppose that $k>3$. Since there exists a pseudo holomorphic line $L$ passing through $P$, whose tangent space at $P$ is the $u$-axis [i]i]. If the almost complex structure is integrable near $P$, the local intersection number of $L$ and $D$ at $P$ is $k>3$. This contradicts the fact that $D \cdot L=3$.

q.e.d.

Now, to prove Theorem $5 . \overline{5}, 1$, we recall the following elementary fact.

Lemma 5.11. Let $S_{1}(t), \ldots, S_{k}(t)$ be smooth one-parameter families of closed embedded submanifolds in a smooth compact manifold $M$. Suppose that $S_{i}(t)$ and $S_{j}(t)$ have at most transversal double points for $i \neq j$. Then, there exists an ambient isotopy $\Psi_{t}$ on $M$ such that $S_{j}(t)=\Psi_{t}\left(S_{j}(0)\right)$ for $j=1, \ldots, k$.

Proof of Theorem $\mathbf{1 5}$. We take a one-parameter family $\left\{J_{t}\right\}$ of $\omega$-tame almost complex structures joining $J_{0}$ and $J_{1}$ such that $J_{t}$ is integrable near the cusp point $p$ for each $t$. Then, for such a family $\left\{J_{t}\right\}$, Theorem s. 5 shows that there is a one-parameter family of singular pseudoholomorphic cubic curves $D_{t}$ joining $D_{0}$ and $D_{1}$ such that the singular points on $D_{t}$ are the fixed point $p$ in $\mathbf{C} P^{2}$. 
We blow-up $\mathbf{C} P^{2}$ at $p$. Since $J_{t}$ is integrable near the point $p$, the $J_{t}$-holomorphic curves $D_{t}$ can be lifted to pseudo holomorphic curves $D_{t}^{\prime}$ for some almost complex structure $\widetilde{J}_{t}$, which are integrable in a neighborhood of the exceptional divisor $E_{t}$. Note that $E_{t}$ and $D_{t}^{\prime}$ are tangents to each other. After making a blow-up at the point $p^{\prime}$, where $E_{t}$ and $D_{t}^{\prime}$ are tangents to each other, the proper transforms of those curves intersect transversely at a point $p^{\prime \prime}$. Then, we get a configuration of four embedded spheres intersecting each other transversely with at most double points. We denote by $\widetilde{D}_{t}$ the proper transform of $D_{t}$, and $E_{1, t}, E_{2, t}$ and $E_{3, t}$ are (proper transforms of) exceptional divisors with self-intersection number $-1,-2$ and -3 , respectively.

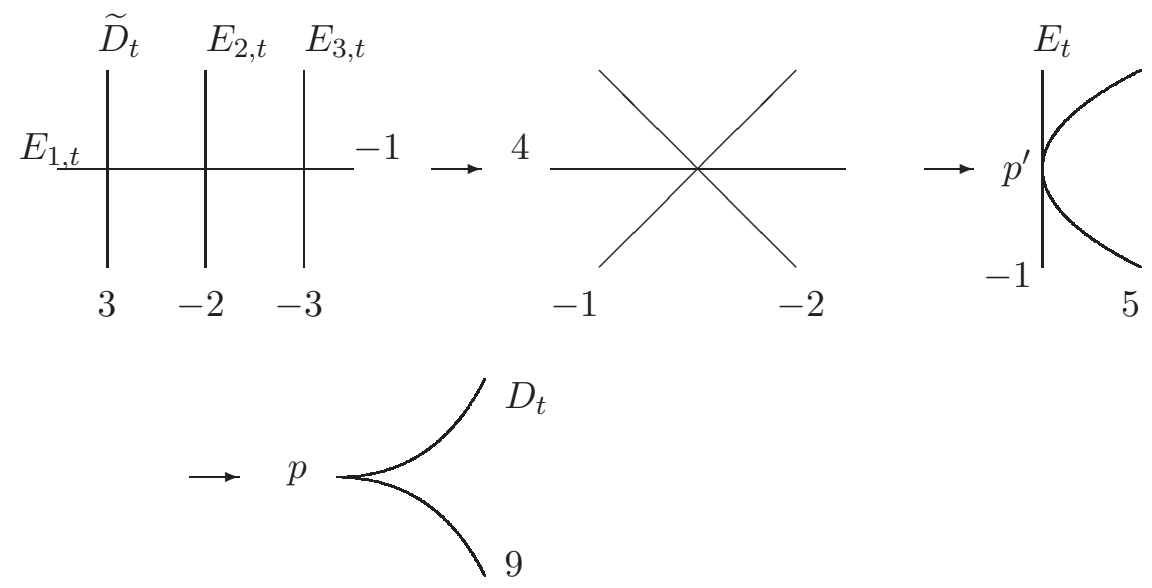

Figure 8.

Note that $E_{1, t}, E_{2, t}$ and $E_{3, t}$ depend smoothly on $t$. Therefore, by Lemma $5 . \overline{1}$, we can find an isotopy $\Psi_{t}$ of the three times blown-up manifold $\vec{M}$ such that $\Psi_{t}\left(\widetilde{D}_{0}\right)=\widetilde{D}_{t}$ and $\Psi_{t}\left(E_{i, 0}\right)=E_{i, t},(i=1,2,3)$. Restricting $\left(\Psi_{t}\right)^{-1}$ to $\widetilde{M} \backslash\left(\widetilde{D}_{t} \cup E_{1, t} \cup E_{2, t} \cup E_{3, t}\right)$, we get a one parameter family of diffeomorphisms from $\mathbf{C} P^{2} \backslash D_{0}$ to $\mathbf{C} P^{2} \backslash D_{t}$. This proves Theorem

q.e.d.

In the proof above, we actually proved the following:

Theorem 5.12. Let $\widetilde{M}, \widetilde{D}_{t}$ and $E_{i, t}$ be as in the proof of Theorem $\overline{5} . \hat{H}_{1}$. Then, the pair $\left(\widetilde{M}, \widetilde{D}_{1} \cup E_{1,1} \cup E_{2,1} \cup E_{3,1}\right)$ is ambient isotopic to $\left(\bar{M}, \widetilde{D}_{0} \cup E_{1,0} \cup E_{2,0} \cup E_{3,0}\right)$. Moreover, there exists a one-parameter 
family $\left\{\omega_{t}\right\}$ of symplectic forms such that $\widetilde{D}_{t}, E_{1, t}, E_{2, t}$ and $E_{3, t}$ are symplectic submanifolds with respect to $\omega_{t}$.

Proof. We only need to show the last statement. Note that the almost complex structures $J_{t}$ are integrable around $p$ and $E_{1, t} \cup E_{2, t} \cup E_{3, t}$ is the exceptional divisor of the three times blow-up in the sense of complex geometry. We can construct the symplectic form $\omega_{t}$ compatible with $\widetilde{J}_{t}$ as in [2-2in]

q.e.d.

Next, we will prove Theorem 5.2 . In the case of $\mathbf{C} P^{1} \times \mathbf{C} P^{1} \backslash D_{1}^{\prime}$, we similarly blow up $\mathbf{C} P^{1} \times \mathbf{C} P^{\mathrm{I}}$ three times to get a configuration of four embedded spheres intersecting each other transversely with at most double points. We denote by $\widetilde{D}_{1}^{\prime}$ the proper transform of $D_{1}^{\prime}$, and by $E_{1}^{\prime}, E_{2}^{\prime}$ and $E_{3}^{\prime}$ the (proper transforms of) exceptional divisors with selfintersection number $-1,-2$ and -3 , respectively. The self-intersection number of $\widetilde{D}_{1}^{\prime}$ is 2 in this case. (See Figure $\bar{p}_{1}^{\prime}$ below.) In addition, we consider pseudo holomorphic rational curves $A$ and $B$ in $\mathbf{C} P^{1} \times \mathbf{C} P^{1}$ which represent the homology classes $\mathbf{C} P^{1} \times\{p t\}$ and $\{p t\} \times \mathbf{C} P^{1}$, respectively, and pass through the $(2,3)$-cusp point $q$. We denote by $\widetilde{A}$ and $\widetilde{B}$ the proper transforms of $A$ and $B$, respectively.

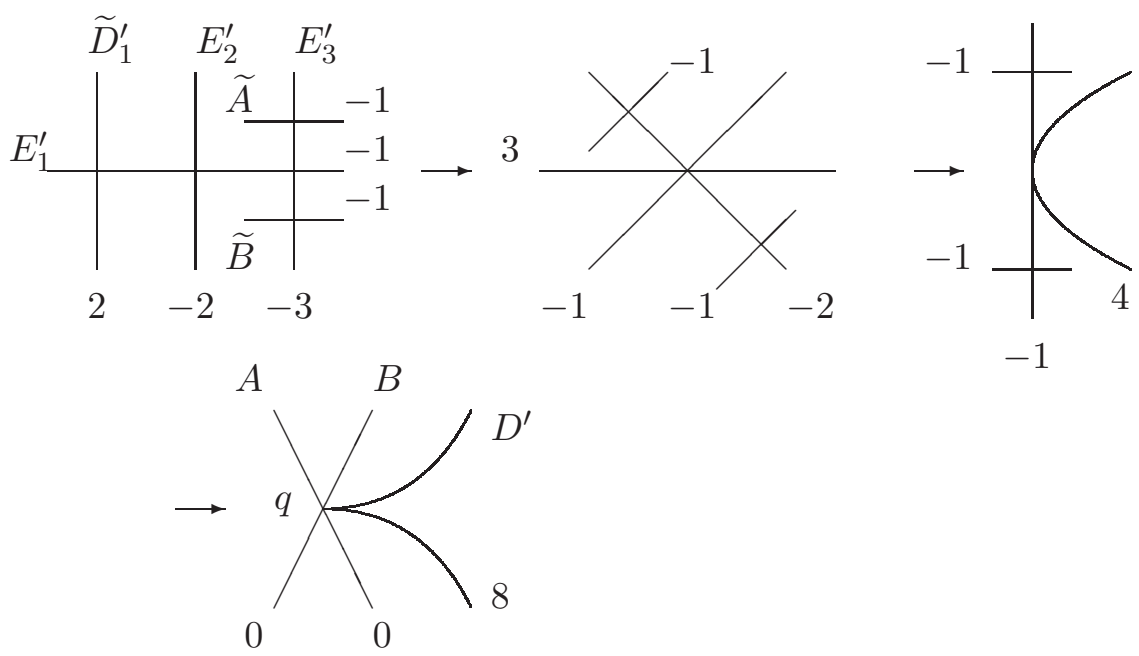

Figure 9. 
Theorem 5.13. Let $\widetilde{M^{\prime}}$ be the three times blown-up manifold of $\mathbf{C} P^{1} \times \mathbf{C} P^{1}$, and $\widetilde{D}_{1}^{\prime}, E_{i}^{\prime}(i=1,2,3)$ and $\widetilde{A}, \widetilde{B}$ as above. Then the ambient isotopy type of the pair $\left(\widetilde{M}^{\prime}, \widetilde{D}_{1}^{\prime} \cup E_{1}^{\prime} \cup E_{2}^{\prime} \cup E_{3}^{\prime} \cup \widetilde{A} \cup \widetilde{B}\right)$ is uniquely determined. Moreover, the ambient isotopy can be chosen in such a way that the divisors $\widetilde{D}_{1}^{\prime}, E_{1}^{\prime}, E_{2}^{\prime}, E_{3}^{\prime}, \widetilde{A}$, and $\widetilde{B}$ are symplectic submanifolds with respect to a certain one-parameter family of symplectic structures.

Proof. First of all, we modify the almost complex structure $J_{1}$ away from $D_{1}^{\prime}$ so that $J_{1}$ is integrable in a neighborhood $U$ of $A \cup B$ and $D_{1}^{\prime} \cup A \cup B$ is $J_{1}$-holomorphic. (By abuse of notations, we use the same $J_{1}$.) Pick a point $r$ on a non-singular part of $D_{1}^{\prime} \cap U$ and denote by $f_{1}$ and $f_{2}$ the fibers, passing through $r$, with respect to the two ruling structures on the product $\mathbf{C} P^{1} \times \mathbf{C} P^{1}$. We blow up $\mathbf{C} P^{1} \times \mathbf{C} P^{1}$ at $r$ and blow down the proper transforms $\tilde{f}_{1}$ and $\tilde{f}_{2}$ of $f_{1}$ and $f_{2}$. From now on, we pick the point $r$ in a small neighborhood of the cusp point $q$ of $D_{1}^{\prime}$ in $\mathbf{C} P^{1} \times \mathbf{C} P^{1}$ so that $f_{1}$ and $f_{2}$ are contained in $U$ and the other intersection point $p_{1}^{\prime}$ (resp. $p_{2}^{\prime}$ ) of $f_{1}$ (resp. $f_{2}$ ) with $D_{1}^{\prime}$ is also close to $q$. In particular, we can blow down $\widetilde{f}_{1}$ and $\widetilde{f}_{2}$ in complex category. Denote by $p_{1}$ and $p_{2}$ the contracted images of $\widetilde{f}_{1}$ and $\widetilde{f}_{2}$, respectively. (Note that $p_{1}$ (resp. $p_{2}$ ) is the image of $p_{1}^{\prime}$ (resp. $p_{2}^{\prime}$ ) under the birational transformation.) Then, we get $\mathbf{C} P^{2}$ with a pseudo-holomorphic cuspidal cubic curve $D_{1}^{\prime \prime}$ with one $(2,3)$-cusp point $p$. By Theorem there is a one-parameter family of $J_{t}$-holomorphic cuspidal cubic curves $D_{t}^{\prime \prime}$ joining $D_{0}^{\prime \prime}=D_{0}$ and $D_{1}^{\prime \prime}$, such that the cusp point on $D_{t}^{\prime \prime}$ is the fixed point $p$ in $\mathbf{C} P^{2}$. Here, $D_{0}$ is a cuspidal cubic curve with respect to the standard complex structure. We pick a one-parameter family of two points $p_{1, t}$ and $p_{2, t}$ on $D_{t}^{\prime \prime}$ such that $p_{i, 1}=p_{i},(i=1,2)$, and $p_{1, t}$ and $p_{2, t}$ are contained in a neighborhood of $p$, where the almost complex structures $J_{t}$ are integrable. We blow-up $\mathbf{C} P^{2}$ at $p_{1, t}$ and $p_{2, t}$. Then, the proper transforms of the degree one $J_{t}$-holomorphic curves passing through $p_{1, t}$ and $p_{2, t}$ are pseudo-holomorphic $(-1)$-curves. We blow-down them to get $\mathbf{C} P^{1} \times \mathbf{C} P^{1}$ and the one parameter family $D_{t}^{\prime \prime}$ is transformed to a one parameter family of $J_{t}^{\prime}$-holomorphic curves $D_{t}^{\prime}$ for some one parameter family $J_{t}^{\prime}$. Here, we used Lemma

Denote by $A_{t}$ and $B_{t}$ the $J_{t}^{\prime}$-holomorphic rational curves passing through $q$ representing the class $[A]$ and $[B]$, which depend smoothly on $t$. After blowing-up $\mathbf{C} P^{1} \times \mathbf{C} P^{1}$ successively three times (as in the proof of Theorem $\overline{5}^{1}$ ), we get smooth one parameter families of embedded surfaces $\widetilde{D}_{t}^{\prime}, \widetilde{E}_{i, t}^{\prime},(i=1,2,3), \widetilde{A}_{t}$ and $\widetilde{B}_{t}$ in $\widetilde{M}^{\prime}$. Note that the homological intersection number of $D_{t}^{\prime}$ and $A_{t}$ (resp. $B_{t}$ ) is 2 and $q$ is a $(2,3)$-cusp point on $D_{t}^{\prime}$ (Lemma $\left.\widetilde{5}_{2}^{-1} \overline{1}_{-1}^{0}\right)$. Hence, for each $t, \widetilde{A}_{t}$ and $\widetilde{B}_{t}$ 
intersect $\widetilde{E}_{3, t}^{\prime}$ transversely and are disjoint from $\widetilde{D}_{t}^{\prime}, \widetilde{E}_{1, t}^{\prime}$ and $\widetilde{E}_{2, t}^{\prime}$. In other words, the configuration as in Figure 99 moves smoothly in $t$ preserving the intersection pattern. Thus, the first part of Theorem 5.13i follows from Lemma Theorem 5.12.

q.e.d.

Since the complement of $\widetilde{D}_{1}^{\prime} \cup E_{1}^{\prime} \cup E_{2}^{\prime} \cup E_{3}^{\prime} \cup \widetilde{A} \cup \widetilde{B}$ (resp. $\widetilde{D}_{1}^{\prime} \cup E_{1}^{\prime} \cup E_{2}^{\prime} \cup$ $\left.E_{3}^{\prime} \cup \widetilde{A}, \widetilde{D}_{1}^{\prime} \cup E_{1}^{\prime} \cup E_{2}^{\prime} \cup E_{3}^{\prime}\right)$ in $\widetilde{M}^{\prime}$ is diffeomorphic to the complement of $D_{1}^{\prime} \cup A \cup B$ (resp. $\left.D_{1}^{\prime} \cup A, D_{1}^{\prime}\right)$ in $\mathbf{C} P^{1} \times \mathbf{C} P^{1}$, we obtain the following:

\section{Corollary 5.14.}

(1) The diffeomorphism type of the complement of $D_{1}^{\prime} \cup A \cup B$ in $\mathbf{C} P^{1} \times \mathbf{C} P^{1}$ is uniquely determined.

(2) The diffeomorphism type of the complement of $D_{1}^{\prime} \cup A$ in $\mathbf{C} P^{1} \times$ $\mathbf{C} P^{1}$ is also uniquely determined.

(3) The diffeomorphism type of the complement of $D_{1}^{\prime}$ in $\mathbf{C} P^{1} \times \mathbf{C} P^{1}$ is also uniquely determined.

Corollary 5.13$)$ is nothing but Theorem

5.3. An alternative proof of Theorem $5 . \overline{1}_{1}^{1}$. When we proved Theorem , in Section 5.2 , we used three times blow-up operations to get the configuration of four rational curves in Figure problem to Lemma 5 . But we can prove Theorem by using a special feature of cuspidal curves with $(2, \overline{3})$-cusps instead of considering blow-ups. (We will also use Theorem here.) Firstly, we recall the following proposition, which is well-known in singularity theory. For the sake of convenience, we give a proof of it.

Proposition 5.15. The holomorphic map germ of $z \mapsto(u, v)=$ $\left(z^{2}, z^{3}\right)+O(4)$ is right-left equivalent to $z \mapsto(u, v)=\left(z^{2}, z^{3}\right)$.

Proof. Write

$$
u(z)=\sum_{i \geq 2} a_{i} z^{i}, v(z)=\sum_{i \geq 3} b_{i} z^{i}
$$

with $a_{2}=1$ and $b_{3}=1$. By a suitable coordinate change of $z$ variable in the domain at germ level, we may assume that $u(z)=z^{2}$. Next, we take new coordinates of the target by

$$
\left(u_{1}, v_{1}\right)=\left(u, v-\sum_{k \geq 2} b_{2 k} u^{k}\right)
$$


so that the map germ is transformed to

$$
\left(u_{1}(z), v_{1}(z)\right)=\left(z^{2}, \sum_{k \geq 1} b_{2 k+1} z^{2 k+1}\right) .
$$

Consider a function defined by

$$
g(z)=\left\{\sum_{k \geq 2} b_{2 k+1} z^{2 k+1}\right\} /\left\{\sum_{k \geq 1} b_{2 k+1} z^{2 k+1}\right\} .
$$

Note that $g(z)$ is an even function. Hence, we can write $g(z)=h\left(z^{2}\right)$ with some holomorphic function $h$ of one variable. Since $b_{3} \neq 0$, which implies that $g$ is a germ of a holomorphic function such that $g(0)=0$. Now, we make another coordinate change of the target by

$$
\left(u_{2}, v_{2}\right)=\left(u_{1}, v_{1}\left(1-h\left(u_{1}\right)\right)\right) .
$$

Then, the map germ is transformed to $\left(u_{2}, v_{2}\right)=\left(z^{2}, z^{3}\right)$.

q.e.d.

Remark 5.16. For a $C^{\infty}$-one parameter family of map germs with $(2,3)$-cusp singularities, the argument above can produce a germ of an ambient isotopy of $\left(\mathbf{C}^{2}, O\right)$ so that the deformation of a $(2,3)$-cusp curve extends to an isotopy in a neighborhood of the origin.

We show the following:

Theorem 5.17. Let $D_{1}$ be a pseudo-holomorphic cuspidal cubic curve in $\mathbf{C} P^{2}$ as in Theorem 15 and $D_{0}$ a singular rational cubic curve defined by $x^{3}+y^{2} z=0$. Then, the pair $\left(\mathbf{C} P^{2}, D_{1}\right)$ is ambient isotopic to the pair $\left(\mathbf{C} P^{2}, D_{0}\right)$.

Proof. By Theorem s.5, there is a one-parameter family of singular pseudo-holomorphic curves $D_{t}$ joining $D_{0}$ and $D_{1}$. We may assume that the singular points are fixed at $p$, and that the almost complex structures are integrable near $p$. Lemma 5.10 guarantees that each $D_{t}$ is a pseudo-holomorphic cuspidal cubic curve. By Proposition 5.15 and Remark $5 . \overline{1}$, there is a one-parameter family of holomorphic embeddings $\phi_{t}$ of a ball $B$ in $\mathbf{C}^{2}$ so that the germ of $D_{t}$ at $p$ is described as $\phi_{t}\left(B, D_{0}^{\prime} \cap B\right)$, where $D_{0}^{\prime}$ is the affine part of $D_{0}$, i.e., $\left\{x^{2}+y^{3}=0\right\}$. In other words, there is a family of neighborhoods $B_{t}=\phi_{t}(B)$ of $p$ and a family of local diffeomorphisms $\psi_{t}=\phi_{t} \circ \phi_{1}^{-1}$ so that $\psi_{t}$ maps $\left(B_{1}, D_{1} \cap B_{1}\right)$ to $\left(B_{t}, D_{t} \cap B_{t}\right)$ diffeomorphically. Pick a family of collar neighborhoods $U_{t}$ of $\partial B_{t}$. Write $B_{t}^{\prime}=B_{t} \backslash U_{t}$. Since $\left\{D_{t} \backslash D_{t} \cap B_{t}^{\prime}\right\}$ is a family of embedded surfaces with boundary, we can extend $\left.\psi_{t}\right|_{U_{t}}$ to an ambient isotopy $\rho_{t}: \mathbf{C} P^{2} \backslash B_{1}^{\prime} \rightarrow \mathbf{C} P^{2} \backslash B_{t}^{\prime}$ so that $\psi_{t}$ and $\rho_{t}$ are 
glued to an ambient isotopy $f_{t}$ of $\mathbf{C} P^{2}$ satisfying $f_{t}\left(D_{1}\right)=D_{t}$. Thus, the pairs $\left(\mathbf{C} P^{2}, D_{1}\right)$ and $\left(\mathbf{C} P^{2}, D_{0}\right)$ are ambient isotopic. $\quad$ q.e.d.

\section{Proof of Main Theorem}

Recall that the minimal symplectic filling is the complement of $\widetilde{C} \cup$ $E_{p} \cup E_{q} \cup E_{r}$ in $Z(\Gamma)$. Therefore, it is diffeomorphic to the complement of a regular neighborhood of $E_{r}^{\prime \prime \prime} \cup A \cup B, E_{r}^{\prime \prime \prime} \cup A, E_{r}^{\prime \prime \prime}$, in $Z\left(A_{n}\right)_{(3)}^{(2)}, Z\left(D_{n}\right)_{(3)}^{(1)}$ and $Z\left(E_{n}\right)_{(3)}$, respectively. After these stages, we started contracting a disjoint family of $(-1)$-curves $\left\{\epsilon_{i}\right\}$. By Lemma 4.1 , we modified the tame almost complex structure so that it is integrable around any $\epsilon_{i}$. Let $p_{i}$ be the contracted image of $\epsilon_{i}$ by the complex blow-down, see Proposition ( $A_{n}$-case).

Firstly, we consider the case of type $E_{n}$. Write $D_{1}=\pi\left(E_{r}^{\prime \prime \prime}\right) \subset \mathbf{C} P^{2}$ in Proposition 4 . As in the proof of Theorem times to get a closed symplectic 4 -manifold $\widetilde{M}$ and the proper transform $\widetilde{D}_{1}$ of $D_{1}$. Denote by $\widetilde{p_{i}}$ on $\widetilde{D_{1}}$ corresponding to $p_{i}$. We choose smooth families $\widetilde{p}_{i}(t)$ on $\widetilde{D_{t}} \backslash\{p\}$, which are mutually disjoint. By modifying the smooth family of tame almost complex structures $J_{t}$ around $\widetilde{p}_{i}(t)$, we may assume that $J_{t}$ is integrable in a neighborhood of $\widetilde{p}_{i}(t)$, while keeping that $\widetilde{D_{t}}$ is $J_{t}$-holomorphic. We blow-up $\left(\widetilde{M}, J_{t}\right)$ at $\widetilde{p}_{i}(t)$ in complex sense to get a smooth family of closed symplectic 4-manifolds $\widetilde{Z}_{t}$. When $t=1$, we find that $\widetilde{Z}_{1}$ is symplectic deformation equivalent to $Z\left(E_{n}\right)$. When $t=0, \widetilde{Z}_{0}$ is nothing but Saito's compactification $\widetilde{M}_{\Gamma}$ of the Milnor fiber, where $\Gamma=E_{n}$, see Section $\underset{2}{\overline{2}}$. Applying Lemma $5 . \overline{1} \overline{1}$ i to the proper transform of $\widetilde{D}_{t} \cup E_{1, t} \cup E_{2, t} \cup E_{3, t}$ (see Theorem 15.12i), we get an isotopy of the pair of $Z\left(E_{n}\right)$ and $\widetilde{C} \cup E_{p} \cup E_{q} \cup E_{r}$ to $\widetilde{M}_{E_{n}}$ and the compactifying divisor arising from the compactification of the Milnor fiber (Figure $\left.\underline{I}_{1}^{\bar{I}}\right)$. This proves Main Theorem for the case of type $E_{n}$.

In the case of type $A_{n}$ (resp. $D_{n}$ ), we contract $\widetilde{A}_{t}$ and $\widetilde{B}_{t}$ (resp. $\left.\widetilde{A}_{t}\right)$ in $\widetilde{Z}$ to recover $Z\left(A_{n}\right)$ (resp. $Z\left(D_{n}\right)$ ). The rest of the argument goes in a similar way using Theorem 5 Theorem 5.12 .

Finally, we prove uniqueness of the symplectic deformation type of $X$. In the proof up to now, we made various choices and perturbations. We will note that the symplectic deformation type does not depend on them.

The symplectic blow-down operation depends on the choice of the normal form around the symplectic $(-1)$-curve, but the symplectic diffeomorphism type, hence, the symplectic deformation type does not 
depend on such a choice. Although the symplectic blow-up operation depends on the choice of the Darboux chart and the radius of the Darboux ball, the symplectic deformation type does not depend on these choices. Hence, the symplectic deformation type of $Z(\Gamma)$ is uniquely

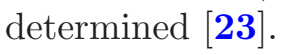

We have shown that $\left(Z(\Gamma), \widetilde{C} \cup E_{p} \cup E_{q} \cup E_{r}\right)$ is isotopic to the standard model, i.e., the compactification of the Milnor fiber and the compactifying divisor. We firstly consider the case that $\Gamma=E_{n}$. In general, they are not necessarily ambient symplectically isotopic, but $\Psi_{t}\left(\widetilde{C} \cup E_{p} \cup E_{q} \cup E_{r}\right)$ is non-degenerate with respect to the symplectic structure. Here, $\Psi_{t}$ is the isotopy in the proof of Theorems 5.12. A similar statement holds in the case that $\Gamma=A_{n}, D_{n}$, based on Theorem 5.13 instead of Theorem 5.12 The last task is to show that their regular neighborhoods are chosen so that the boundary is always symplectically concave. For this purpose, we choose the regular neighborhood as follows. We contract $E_{p}, E_{q}$ and $E_{r}$ to cyclic quotient singularities. The ambient space is a $V$-manifold with an almost complex structure, which is integrable near the image $D$ of $\widetilde{C}$. Pick a Hermitian metric near $D$ and denote by $N(D)$ a tubular neighborhood of $D$ with the radius $\delta>0$. Since the Euler number of the normal bundle of $D$, as a complex $V$-vector bundle, is positive, the boundary of the disk bundle is symplectically concave. The inverse image of $N(D)$ by the contraction map is a regular neighborhood of the compactifying divisor. We can use $N(D)$ as the strong concave filling of the link $L$. This argument works also for the image of divisors under the isotopy. Note also that if we change the radius of the tubular neighborhood, we get a smooth family of symplectic forms on $X$ with respect to which the boundary $\partial X$ is strongly symplectically convex. Therefore, we get uniqueness of the symplectic deformation type of $X$.

Remark 6.1. We treat cases of $A_{n}, D_{n}$ and $E_{n}$ in a similar way based on Theorem 5.1 and Corollary 15.14 . Here, we would like to comment other approaches for cases of $A_{n}^{-}$and $D_{n}$. For $A_{n}$-case, we can contract a maximal family of symplectic $(-1)$-curves in $Z\left(A_{n}\right)_{(2)}$, which are disjoint from $E_{p}^{\prime \prime}$ to get the complex projective plane. The compactifying divisor is transformed to the union of a pseudo holomorphic line and a pseudo holomorphic conic, which are tangential to each other. For $D_{n}$ case, we can contract a maximal family of symplectic $(-1)$-curves in $Z\left(D_{n}\right)_{(2)}$, which are disjoint from $E_{p}^{\prime \prime}$ to get the product of two copies of the complex projective line. The compactifying divisor is transformed to the union of two pseudo holomorphic spheres of bidegree $(1,0)$ and $(1,2)$, 
respectively, which are tangential to each other. The rest of the argument is based on uniqueness of isotopy type of these divisors in the projective plane or the product of two copies of the complex projective line.

\section{Acknowledgment}

This work was completed during both authors' stay at the MaxPlanck-Institut für Mathematik, Bonn. They would like to express gratitude to the institute for nice environment and hospitality. The second author wishes to thank the Max-Planck-Institut for financial support. They would also like to thank Masa-Hiko Saito, who suggested to them some simplification of the argument in an early stage of this work, and Goo Ishikawa for useful information. Thanks are also due to the anonymous referee, whose comments improved the exposition.

\section{References}

[1] J.-F. Barraud, Nodal symplectic spheres in $\mathbf{C P}^{2}$ with positive self intersection, Internat. Math. Res. Notices 9 (1999) 495-508, MR 1692591. (2000f:57026), $\mathrm{Zbl}$

[2] J.-F. Barraud, Coubes pseudo-holomorphes equisingulaieres en dimension 4, Bull. Soc. Math. France 128 (2000) 179-206, MR 1772440 (2001i:53154), Zbl 0976.53037

[3] E. Brieskorn, Über die Auflösung gewisser Singularitäten von holomorphen Abbildungen, Math. Ann. 166 (1966) 76-102, MR 0206973 (34 \#6789), $\mathrm{Zbl}$

[4] E. Brieskorn, Die Auflösung der rationalen Singularitäten holomorpher Abbildungen, Math. Ann. 178 (1968) 255-270, MR 0233819. (38 \#2140), Zbl $0 \overline{159}-\overline{3} 7 \overline{7} 03$

[5] E. Brieskorn, Singular elements of semi-simple algebraic groups, Proc. Internat. Congress Math. Nice, 1970, 279-284, MR 0437798 (55 \#10720), Zbl 0223.22012.

[6] J.W. Bruce \& T.J. Gaffney, Simple singularities of mappings _. J. London _ Math. Soc. 26 (1982) 465-474, MR 10684560 (84d:32012), Zbl 0575.58008

[7] Y. Eliashberg, On symplectic manifolds_with some contact properties,__._. Differential Geom. 33 (1991) 233-238, MR 1085141 (92g:57036), Zbl 0735.53021.

[8] K.A. Froyshov, The Seiberg-Witten equations and four-manifolds with boundary, Math. Res. Lett. 3 (1996) 373-390, MR 1397685 (97i:57037), Zbl 0872.57024.

[9] K. Fukaya \& K. Ono, Arnold conjecture and Gromov-Witten invariant, Topology 38 (1999) 933-1048, MR 1688434 (2000j:53116), Zbl, 0946.53047.

[10] P. Griffiths \& J. Harris, Principle of Algebraic Geometry, Wiley, 1978, MR $0507725 !$ (80b:14001), Zbl 0408.14001 .

[11] M. Gromov, Pseudo holomorphic curves in symplectic manifolds, Invent. Math. 82 (1985) 307-347, MR 0809718 (87j:53053), Zbl 0592.53025. 
[12] H. Hofer, V. Lizan \& J.C. Sikorav, On genericity for holomorphic curves in 4-dimensional almost-complex manifolds, J. Geom. Anal. 7 (1997) 149-159, MR '1630789! (2000d:32045), Zbl 0911.53014.

[13] H. Hofer \& E. Zehnder, Symplectic Invariants and Hamiltonian Dynamics, Birkhäuser _Verlag, Basel, Boston, Berlin, 1994, MR 1306732 (96g:58001), Zbl 0805.58003

[14] S. Ivashkovich \& V. Shevchishin, Structure of moduli space in a neighborhood of a cusp-curve and meromorphic hulls, Invent. Math. 136 (1999) 571-602, MR 1695206i (2001d:32035), Zbl 0930.32017.

[15] Y. Kanda, The monopole equations and J-holomorphic curves on weakly convex almost Kahler 4-manifolds, Trans. Amer. Math. Soc. 353 (2001) 2215-2243, MR '1814068' (2002b:57036), Zbl 0969.57021.

[16] S. Kobayashi \& K Nomizu, Foundations of Differential Geometry, II, WileyInterscience Publ., New York, 1969, MR 0238225 (38 \#6501), Zbl 0175.48504

[17] F. Lalonde \& D. McDuff, The classification of ruled symplectic 4-manifolds, Math. Res. Lett. 3 (1996) 769-778, MR 1_1426534 (98b:57040), Zbl 0874.57019.

[18] H.V. Lê \& K. Ono, Perturbation_of pseudo-holomorphic_curves, Internat. J.

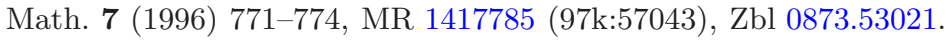

[19] T.J. Li \& A. Liu, Symplectic structure on ruled surfaces and $\underline{\text { a }}$ generalized adjunction formula, Math. Res. Lett. 2 (1995) 453-471, MR 1355707! (96m:57052), $\mathrm{Zbl} 0855.53019$

[20] P. Lisca, Symplectic fillings and positive scalar curvature, Geom. Topol. 2 (1998) 103-116, MR 1633282 (99f:57038), Zbl 0942.53050.

[21] A. Liu, Some new applications of general wall crossing formula, Gompf's conjecture and its applications, Math. Res. Lett. 3 (1996) 569-585, MR 1418572 (97k:57038), Zbl 0872.57025!

[22] D. McDuff, Examples of simply-connected symplectic non-Kählerian manifolds, J. Differential Geom. 20 (1984) 267-277, MR 0772133 (86c:57036), $\mathrm{Zbl} 0567.53031$

[23] D. McDuff, The structure of rational and ruled symplectic 4-manifolds, J. Amer. Math. Soc. 3 (1990) 679-712, MR 168961! (93k:58098), Zbl 0723.53019.

[24] D. McDuff, The local behaviour of holomorphic curves in almost complex 4-manifolds, J. Differential Geom. 34 (1991) 143-164, MR $\overline{1} \overline{1} \overline{1} \overline{4} \overline{5} \bar{\theta}$ (93e:53050), Zbl 0736.53038

[25] M. Namba, Geometry of Projective, Algebraic Curves, Marcel Dekker, Inc. New York and Basel, 1984, MR 0768929 (86d:14021), Zbl 0556.14012.

[26] H. Ohta \& K. Ono, Notes on symplectic 4-manifolds with $\underline{b}_{2}^{+}=\underline{1}_{2} \underline{I I}_{3}$ Internat.

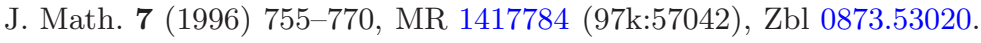

[27] H. Ohta \& K. Ono, Simple singularities and topology of symplectically_ fill-, ing 4-manifolds, Comment. Math. Helv. 74 (1999) 575-590, MR 1730658 (2001b:57060), Zbl $0957.57022 !$

[28] H. Ohta \& K. Ono, Symplectic fillings of the link of_simple elliptic singularities, J. Reine Angew. Math. 565 (2003) 183-205, MR 2024651, Zbl 1044.57008.

[29] H. Ohta \& K. Ono, Symplectic 4-manifolds containing singular rational curves with $(2,3)$-cusp, Séminaires et. Congrès, Soc. Math. France. 10(2005) 233-241. 
[30] M. Oka, Symmetric curves with nodes and_ cusps,_J. Math. Soc. Japan 44 (1992) 375-414, MR 167373 (93m:14024), Zbl 0767.14011!

[31] K. Saito, A new relation among Cartan matrix and_Coxeter matrix, J. Algebra 105 (1987) 149-158, MR 0871750 (88c:14003), Zbl 0613.14003!

[32] K. Saito, Around the theory of the generalized weight system: Relations with singularity theory, the generalized Weyl group and its invariant theory, etc., Amer. Math. Soc. Transl. (2) 183 (1998) 101-143, MR 1615139, Zbl 0622.20040.

[33] B. Siebert and G. Tian, preprint.

[34] J.-C. Sikorav, The gluing construction for normally generic J-holomorphic curves, in "Symplectic and contact topology; interactions and perspectives", 175-199, Fields Institute Communications, 35, Amer. Math. Soc., 2003, MR 1969276" (2004j:32025).

[35] Y.T. Siu \& S.-T. Yau, Compact Kähler manifolds of positive bisectional curvature, Invent. Math. 59 (1980) 189-204, MR , "0577360' (81h:58029), Zbl 0442.53056

[36] O. Zariski, On the problem of existence of algebraic functions of two variables possessing a given branch curve, Amer. J. Math. 51 (1929) 305-328, JFM 55.0806 .01 .

Graduate School of Mathematics

NAGOYA UNIVERSITY

NAGOYA 464-8602

JAPAN

Department of Mathematics

HOKKAIDO UNIVERSITY

SAPPORO 060-0810

JAPAN 